\title{
Identification and therapeutic rescue of autophagosome and glutamate receptor defects in C9ORF72 and sporadic ALS neurons
}

\author{
Yingxiao Shi, ${ }^{1,2,3}$ Shu-Ting Hung, ${ }^{1,2,3}$ Gabriel Rocha, ${ }^{1,2,3}$ Shaoyu Lin, ${ }^{1,2,3}$ Gabriel R. Linares, ${ }^{1,2,3}$ \\ Kim A. Staats, ${ }^{1,2,3}$ Carina Seah, ${ }^{1,2,3}$ Yaoming Wang, ${ }^{3,4}$ Michael Chickering, ${ }^{1,2,3}$ Jesse Lai, 1,2,3 \\ Tohru Sugawara, ${ }^{1,2,3}$ Abhay P. Sagare, , Berislav V. Zlokovic, ${ }^{3,4}$ and Justin K. Ichida ${ }^{1,2,3}$ \\ 'Department of Stem Cell Biology and Regenerative Medicine, Keck School of Medicine, University of Southern California, \\ Los Angeles, California, USA. ${ }^{2}$ Eli and Edythe Broad CIRM Center for Regenerative Medicine and Stem Cell Research at \\ USC, Los Angeles, California, USA. ${ }^{3}$ Zilkha Neurogenetic Institute, Keck School of Medicine of the University of Southern \\ California, Los Angeles, California, USA. ${ }^{4}$ Department of Physiology and Neuroscience, Keck School of Medicine, University \\ of Southern California, Los Angeles, California, USA.
}

Amyotrophic lateral sclerosis (ALS) is a fatal motor neuron disease with diverse etiologies. Therefore, the identification of common disease mechanisms and therapeutics targeting these mechanisms could dramatically improve clinical outcomes. To this end, we developed induced motor neuron (iMN) models from C9ORF72 and sporadic ALS patients to identify targets that are effective against these types of cases, which together comprise approximately $90 \%$ of patients. We find that iMNs from C9ORF72 and several sporadic ALS patients share 2 common defects - impaired autophagosome formation and the aberrant accumulation of glutamate receptors. Moreover, we show that an anticoagulation-deficient form of activated protein C, 3K3A-APC, rescues these defects in both C9ORF72 and sporadic ALS iMNs. As a result, 3K3A-APC treatment lowers C9ORF72 dipeptide-repeat protein (DPR) levels, restores nuclear TDP-43 localization, and rescues the survival of both C9ORF72 and sporadic ALS iMNs. Importantly, 3K3A-APC also lowers glutamate receptor levels and rescues proteostasis in vivo in C9ORF72 gain- and loss-of-function mouse models. Thus, motor neurons from C9ORF72 and at least a subset of sporadic ALS patients share early defects in autophagosome formation and glutamate receptor homeostasis and a single therapeutic approach may be efficacious against these disease processes.

contributed equally to this work.

Conflict of interest: $\mathrm{JKI}$ is a cofounder of AcuraStem Incorporated. JKI declares that he is bound by confidentiality agreements that prevent him from disclosing details of his financial interests in this work. BVZ is a scientific founder and chairs the scientific advisory board of $Z Z$ Biotech LLC, a biotechnology company with a mission to develop activated protein $C$ and its functional mutants for the treatment of stroke and other neurological disorders.

Copyright: (c) 2019, American Society for Clinical Investigation.

Submitted: January 26, 2019

Accepted: June 27, 2019

Published: August 8, 2019.

Reference information: JCl Insight. 2019;4(15):e127736.

https://doi.org/10.1172/jci.

insight.127736.

\section{Introduction}

The genetic etiologies that underlie amyotrophic lateral sclerosis (ALS) are diverse (1). This underscores the need to identify therapeutic targets that are broadly efficacious. The identification of pathophysiological processes that are shared between sporadic ALS patients could enable the development of broadly efficacious therapeutics. Genetic, neuropathological, and pharmacological approaches implicate aberrant proteostasis and neuronal excitability in ALS and other neurodegenerative diseases (2-6), but the mechanisms that drive defective proteostasis and hyper- or hypoexcitability in patient neurons are unclear, and it is unknown if there are therapeutic targets that will be capable of mitigating these processes in multiple different ALS subtypes.

The lack of models for sporadic ALS has hampered efforts to identify disease mechanisms and therapeutic targets that apply broadly to multiple sporadic forms of ALS (3, 7). To address this, we derived induced pluripotent stem cells (iPSCs) from a cohort of sporadic ALS patients and generated induced motor neurons (iMNs) from these iPSCs. We show that similarly to C9ORF72 ALS iMNs, the sporadic ALS iMNs from all 6 donors degenerate significantly faster than controls and show pronounced mislocalization of transactive response DNA-binding protein $43 \mathrm{kDa}$ (TDP-43). Thus, these sporadic ALS iMNs display relevant disease processes.

We hypothesized that phenotypic analysis of iMNs from these sporadic ALS patients and C9ORF72 ALS/frontotemporal dementia (FTD) patients would enable the identification of degenerative mechanisms 
that are shared by C9ORF72 and at least a subset of sporadic ALS motor neurons. We further hypothesized that pharmacologic agents capable of normalizing these phenotypes in C9ORF72 and sporadic ALS iMNs might also rescue neurodegeneration and lead to the identification of therapeutic targets with broad efficacy in the ALS population.

Indeed, we find that C9ORF72 and these sporadic ALS iMNs share defects in autophagosome formation and the aberrant accumulation of glutamate receptors. We find that an engineered, anticoagulation-deficient form of activated protein C called 3K3A-APC, but not heat-inactivated 3K3AAPC (inactive 3K3A-APC), can restore autophagosome formation in both C9ORF72 ALS/FTD and sporadic ALS iMNs. Interestingly, 3K3A-APC also normalizes glutamate receptor levels in C9ORF72 and sporadic ALS iMNs. As a result of these activities, 3K3A-APC lowers dipeptide-repeat protein (DPR) levels in C9ORF72 ALS/FTD iMNs, and potently restores nuclear TDP-43 localization and the normal survival of both C9ORF72 and sporadic ALS iMNs. We show that the ability of 3K3A-APC to rescue ALS iMN survival is dependent on its ability to activate protease-activated receptor 1 (PAR1), identifying PAR1 as a therapeutic target for both C9ORF72 and sporadic ALS. Importantly, 3K3AAPC also rescues glutamate receptor levels and proteostasis impairments in vivo. Thus, motor neurons from C9ORF72 and at least a subset of sporadic ALS patients share common defects in autophagosome formation and glutamate receptor homeostasis, and pharmacologic rescue of these defects by PAR1 activation may slow or prevent neurodegeneration in a substantial fraction of ALS cases.

\section{Results}

Identification of neurodegenerative phenotypes in iMNs from sporadic ALS patients. We and others have shown that iPSC-based models can recapitulate ALS disease processes, provided the iPSC donors harbor ALS-causing genetic variants $(4-6,8-12)$. As a key example, we have shown that iMNs from control lines consistently survive longer than iMNs from C9ORF72 ALS lines ( $n=4$ controls and $n=6$ C9ORF72 ALS patients) (4, 5, $8)$. Because all environmental factors are held constant among the different lines in the iMN survival assay, we reasoned that sporadic ALS patients whose iMNs degenerate significantly faster than control iMNs likely harbored genetic mutations that promoted their observed motor neuron disease. Therefore, these sporadic ALS iMNs could serve as models of sporadic ALS.

To identify sporadic ALS iPSC lines whose iMNs degenerated more rapidly than controls, we generated iPSCs from 6 sporadic ALS patients. We did not bias our selection of sporadic ALS samples based on genetic or clinical information. Whole-genome or -exome sequencing and repeat-primed PCR for the C9ORF72 locus showed that the 6 sporadic ALS patients did not contain rare variants in known ALS genes, nor a C9ORF72 repeat expansion (Supplemental Figure 1, A and B, and Supplemental Tables 1 and 2; supplemental material available online with this article; https://doi.org/10.1172/jci.insight.127736DS1).

To confirm our previous findings showing that iMNs derived from patients harboring ALS-causing genetic mutations display rapid neurodegeneration in vitro $(4,5,8)$, we used transcription factor-mediated conversion $(4,13)$ to generate iMNs from fibroblast-like cells derived from 3 control and 3 C9ORF72 ALS patient iPSC lines that we previously established and characterized (Figure 1A, Supplemental Table 1, and ref. 4). To track individual iMNs, we labeled them using an Hb9::RFP lentivirus that accurately labels HB9+ motor neurons derived in this manner (refs. 4, 5, 8, Figure 1A, and Supplemental Figure 1C) and used robotic microscopy and longitudinal tracking of iMNs to measure their survival (ref. 4 and Figure 1B). The density of $H b 9:: \mathrm{RFP}^{+}$iMNs corresponded to an approximately $10 \%-25 \%$ conversion rate and was similar across the different control and C9ORF72 ALS lines, and iMNs showed consistent survival across the range of iMN densities used in our experiments (Supplemental Figure 1, C-E). The vast majority of neurons in iMN cultures were motor neurons, with only rare tyrosine hydroxylase ${ }^{+}$(dopaminergic neuron marker) or CTIP2+ (cortical neuron marker) neurons detected (Supplemental Figure $1, \mathrm{~F}-\mathrm{I})$. iMN cultures were supplemented with primary mouse mixed glia to facilitate iMN maturation.

Consistent with our previous studies $(4,8)$, a 12-hour pulse treatment of $10 \mu \mathrm{M}$ glutamate induced significantly faster degeneration of C9ORF72 patient iMNs compared with controls (Figure 1, B [individual lines shown] and C [iMNs from all control or patient lines combined into a single trace for clarity], and Supplemental Figure 1, J and K). Glutamate treatment was not strictly required to elicit this effect, as withdrawal of neurotrophic factor supplementation also selectively induced the rapid degeneration of C9ORF72 patient iMNs without glutamate treatment (Figure 1D and Supplemental Figure 1L). Thus, ALS-causing mutations can cause iMNs to degenerate significantly faster than those from controls. 
A

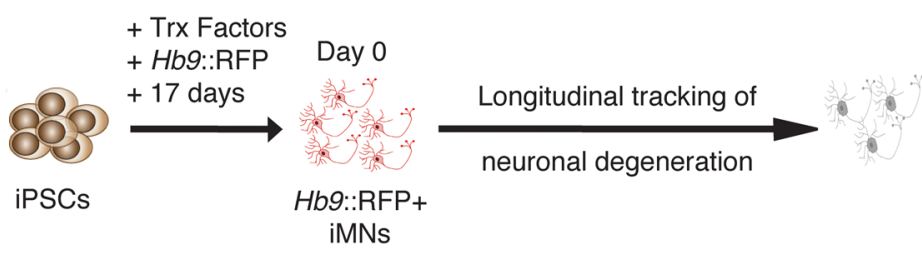

C $\rightarrow-$ CTRL (3 patients, $270 \mathrm{iMNs})$
$\rightarrow-$ C9-ALS (2 patients, $180 \mathrm{iMNs})$ + Glutamate $(10 \mu \mathrm{M})$
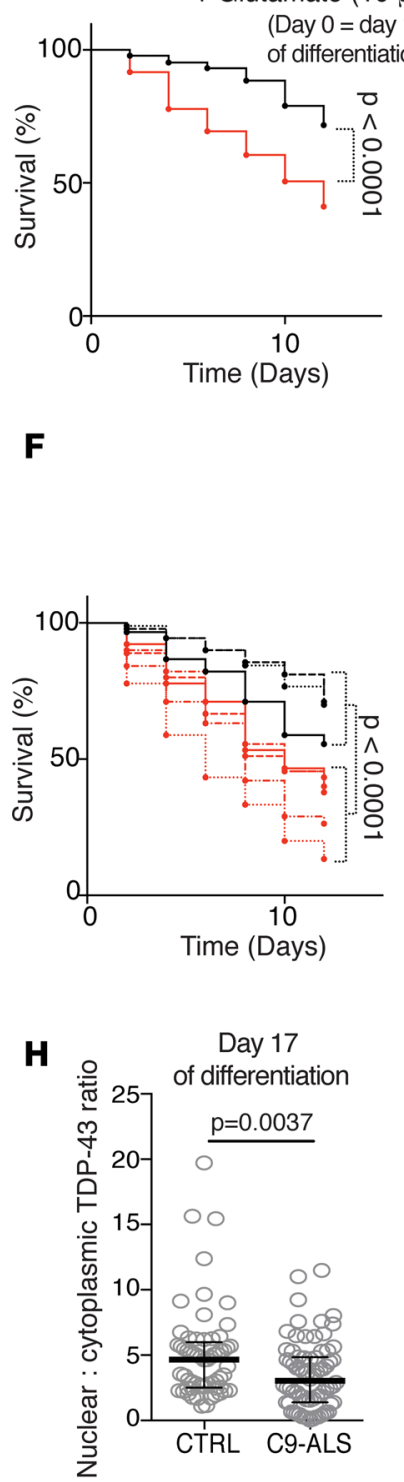

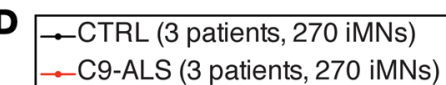

C9-ALS (3 patients, 270 inN

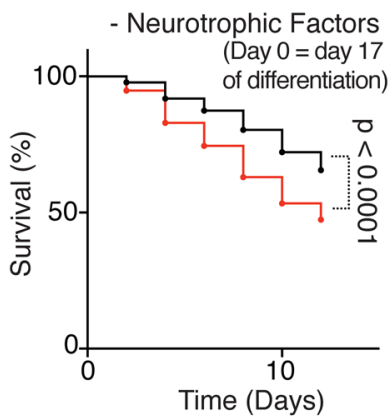

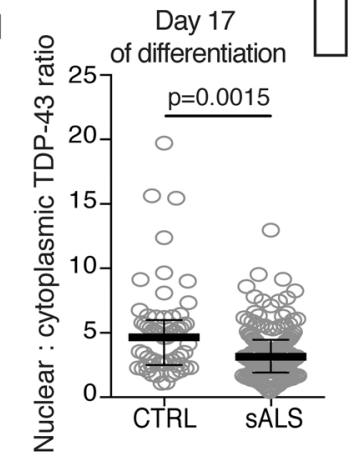

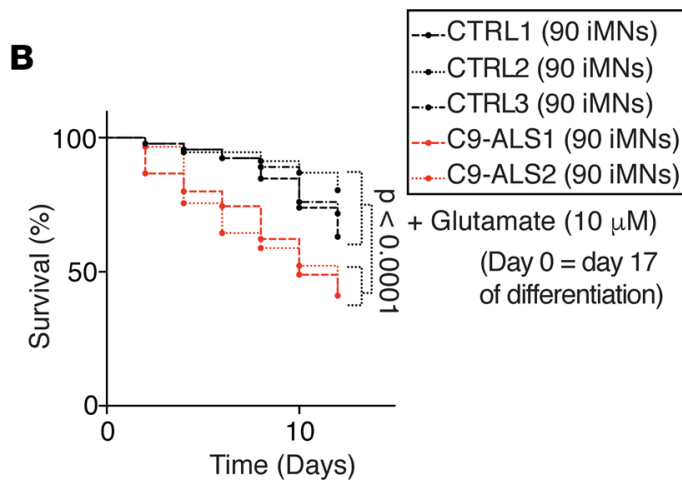

E

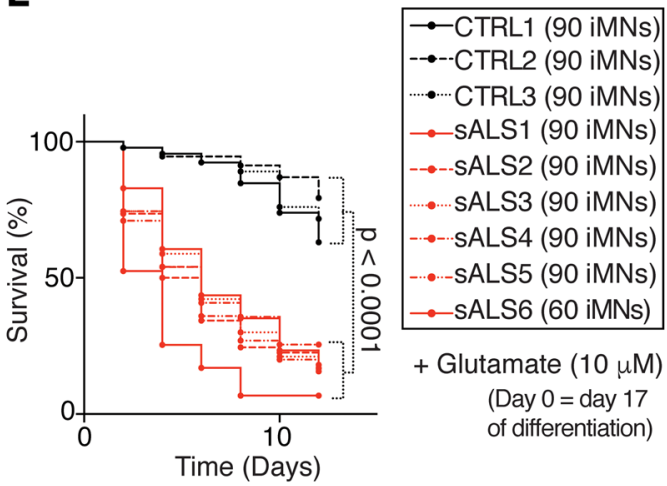

$\mathbf{F}$

H

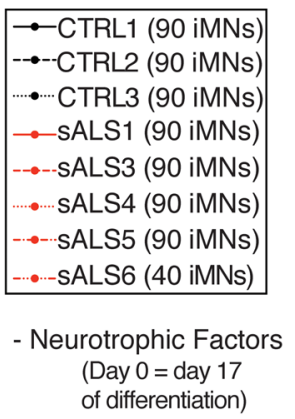

G
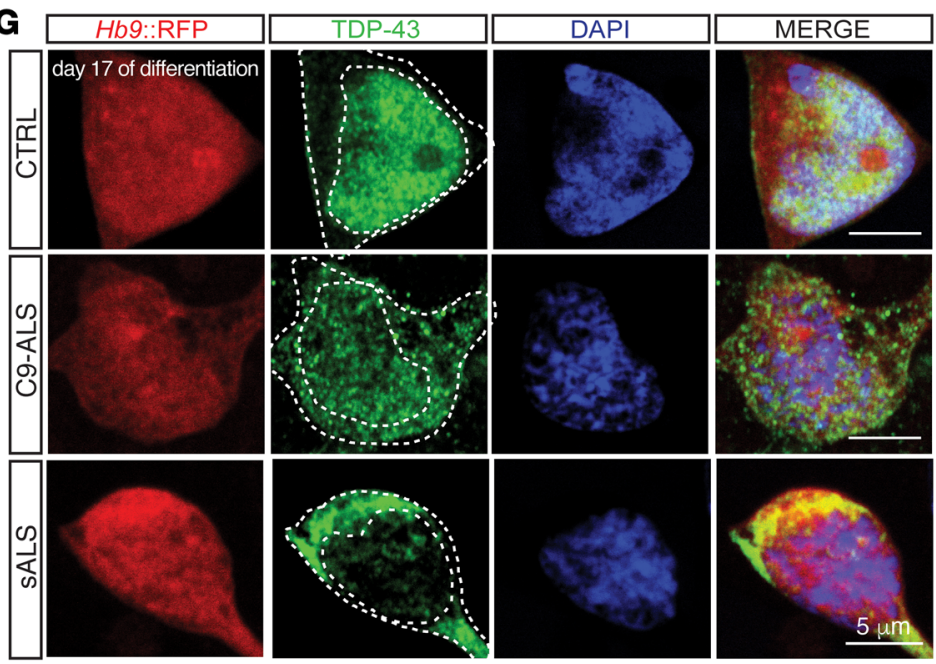

$5 \mu \mathrm{m}$

Figure 1. Identification of neurodegenerative phenotypes in sporadic ALS patient iMNs. (A) Production of $H b 9:: R^{2} P^{+}$iMNs and survival tracking by time-lapse microscopy. (B and C) Survival of control (CTRL) and C9ORF72 ALS patient (C9-ALS) iMNs with a 12-hour pulse treatment of excess glutamate shown for each individual line separately (B), or for iMNs from all lines in aggregate (C). For $\mathbf{B}$ and $\mathbf{C}, n=90$ iMNs per line for 3 control and 2 C9-ALS lines, iMNs quantified from 3 biologically independent iMN conversions per line. (D) Survival of control and C9ORF72 ALS patient iMNs after withdrawal of neurotrophic factor supplementation. iMNs from all control or C9ORF72 patient lines shown in aggregate. $n=90$ iMNs per line for 3 control and 3 C9-ALS lines, iMNs quantified from 3 biologically independent iMN conversions per line. (E and F) Survival of control and sporadic 
ALS (sALS) patient lines after glutamate treatment (E) or withdrawal of neurotrophic factor supplementation (F). iMNs from all control or C9ORF72 patient lines shown in aggregate. $n=90$ iMNs per line for 3 control and 6 (E) or 5 (F) sporadic ALS lines, except sALS6 which had 60 (E) or 40 (F) iMNs counted. iMNs quantified from 3 biologically independent iMN conversions per line. (G-I) Immunofluorescence analysis of total TDP-43 (G) and quantification of the ratio of nuclear to cytoplasmic TDP-43 in control, C9-ALS (H), or sporadic ALS (I) iMNs. Ratio of nuclear to cytoplasmic TDP-43 in individual iMNs treated with $10 \mathrm{nM}$ inactive 3K3A-APC or 3K3A-APC for 6 days. iMNs from 2 controls and 2 C9-ALS patients (H) or 4 sporadic ALS patients (I) were quantified. $n=30$ (controls), 30 (C9-ALS), or 36 (sporadic) iMNs per line per condition from 2 biologically independent iMN conversions of 2 control, 2 C9-ALS, or 4 sporadic ALS lines were quantified. Each gray circle represents a single iMN. Median \pm interquartile range. Unpaired Mann-Whitney test. Scale bars: $5 \mu \mathrm{m}$. Dotted lines outline the nucleus and cell body. For iMN survival experiments, significance was measure by 2-sided log-rank test using the entire survival time course. The day of differentiation stated on each panel indicates the day of differentiation on which the experimental treatment or time course was initiated.

With either pulsatile glutamate treatment or neurotrophic factor withdrawal, iMNs from all 6 sporadic ALS patients degenerated significantly faster than control iMNs (Figure 1, B-F, and Supplemental Figure $1, \mathrm{M}$ and $\mathrm{N}$ ). iMNs derived from additional iPSC clones from the same sporadic ALS patients also degenerated faster than control iMNs (Supplemental Figure 1, O-Q). Thus, iMNs derived from these sporadic ALS patients degenerate significantly faster than controls and at a similar rate to C9ORF72 ALS iMNs. These findings strongly suggest that the donor sporadic ALS patients from which these iMNs were derived harbored genetic variants that contributed to their motor neuron disease. Therefore, iMNs from these patients have the potential to provide insights into mechanisms of neurodegeneration in sporadic ALS cases.

A key molecular hallmark of ALS is the nuclear depletion and cytoplasmic accumulation of TDP-43 in motor neurons (14). The cytosolic accumulation of TDP-43 is thought to drive motor neuron degeneration $(14,15)$. Immunofluorescence verified that both C9ORF72 and sporadic ALS iMNs possessed a lower nuclear-to-cytoplasmic ratio of TDP-43 than controls (Figure 1, G-I; 2 controls, 2 C9ORF72 ALS, and 6 sporadic ALS patients). Thus, these sporadic ALS iMNs exhibit neurodegenerative processes.

C9ORF72 and sporadic ALS iMNs share autophagosome abnormalities that are rescued by $3 K 3 A-A P C$. To identify shared disease mechanisms between C9ORF72 and sporadic ALS motor neurons, we performed phenotypic analysis of patient iMNs. Previous studies have shown that reducing C9ORF72 protein levels using siRNAs impairs autophagosome formation in neurons $(16,17)$. To determine if the reduction in C9ORF72 protein levels caused by the C9ORF72 repeat expansion (4) is sufficient to block autophagosome formation in motor neurons, we expressed an mRFP-GFP-LC3 construct in iMNs to enable quantification of autophagosomes, which are $\mathrm{GFP}^{+}$and $\mathrm{mRFP}^{+}$, whereas autolysosomes are only $\mathrm{mRFP}^{+}$due to the lability of GFP at the acidic $\mathrm{pH}$ of lysosomes (18). Treatment with $50 \mathrm{nM}$ bafilomycin to block autophagosome-lysosome fusion significantly increased the number of $\mathrm{GFP}^{+} \mathrm{mRFP}^{+}$vesicles, indicating that this system could accurately monitor autophagosome number in iMNs (Figure 2A [CTRL, -bafilomycin vs. CTRL, +bafilomycin] and Supplemental Figure 2A; 3 controls). C9ORF72 ALS iMNs contained significantly fewer $\mathrm{GFP}^{+} \mathrm{mRFP}^{+}$vesicles than controls (Figure 2, A and B [CTRL, inactive 3K3A-APC, +bafilomycin vs. C9-ALS, inactive 3K3A-APC, +bafilomycin]; 3 controls and 3 C9ORF72 ALS patients), indicating that autophagosome formation was impaired in C9ORF72 ALS iMNs.

Interestingly, in the presence of $50 \mathrm{nM}$ bafilomycin, sporadic ALS iMNs also contained significantly fewer $\mathrm{GFP}^{+} \mathrm{mRFP}^{+}$vesicles than controls (Figure 2, A and C, and Supplemental Figure 2B; 3 controls and 5 sporadic ALS patients), indicating that autophagosome formation was impaired in sporadic ALS iMNs. Thus, both the C9ORF72 and sporadic ALS iMNs display decreased rates of autophagosome formation.

C9ORF72 regulates autophagy by promoting the RAB1A-dependent trafficking of the ULK1 autophagy initiation complex to the phagophore (16). Consequently, mTOR inhibitors were shown to be ineffective at rescuing autophagosome formation in C9ORF72-deficient neurons and it is unclear if pharmacological agents can restore normal autophagy initiation (16).

In surveying pharmacological agents that might be capable of promoting autophagosome formation in C9ORF72 ALS iMNs, we identified activated protein $\mathrm{C}$ as a possible candidate. Activated protein $\mathrm{C}$ is an endogenous blood serine protease with both anticoagulant and cytoprotective activities, that latter of which is mediated by the activation of PAR1 through non-canonical cleavage (19). Zhong and colleagues previously showed that activated protein C treatment can rescue neurodegeneration in SOD1G93A mice (20) and promote autophagosome formation in murine lung epithelial cells (21). However, it remained unclear if activated protein $C$ could stimulate autophagosome formation in C9ORF72 ALS iMNs, rescue neurodegeneration in non-SOD1 forms of ALS, or rescue neurodegenerative processes in patient-derived motor neurons. To test these possibilities, we used 3K3A-APC, an engineered form of activated protein C 
A Day 17 of differentiation

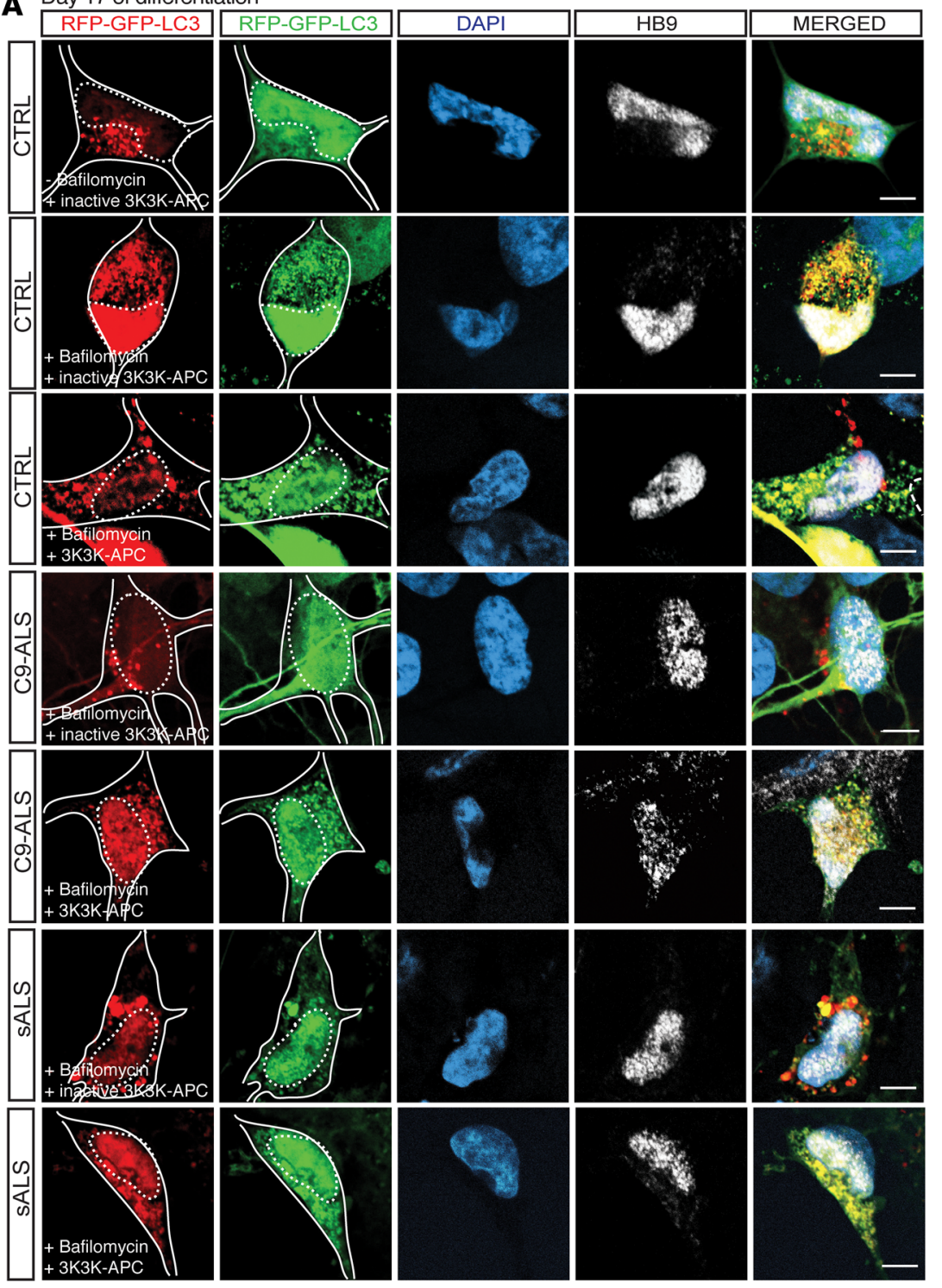

E

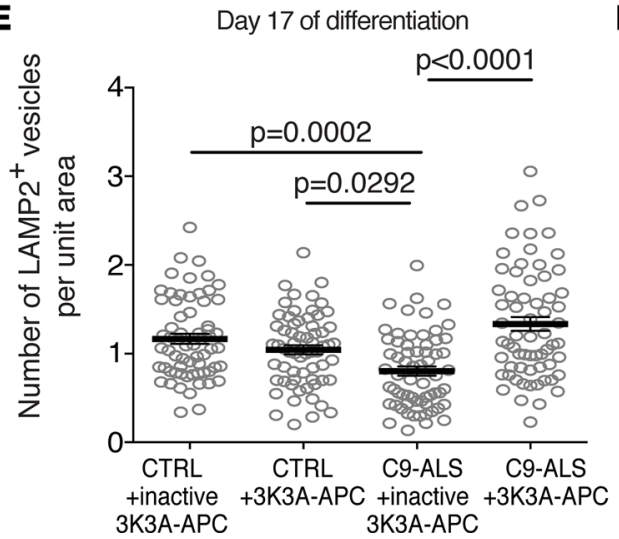

B
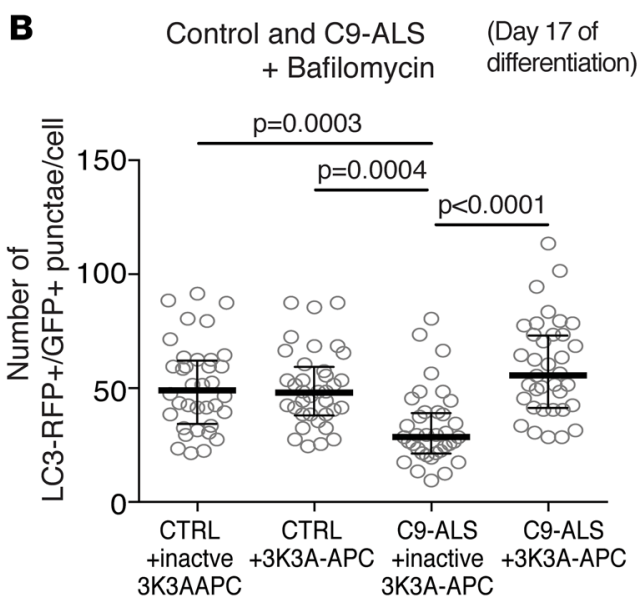

C Control and sALS iMNs

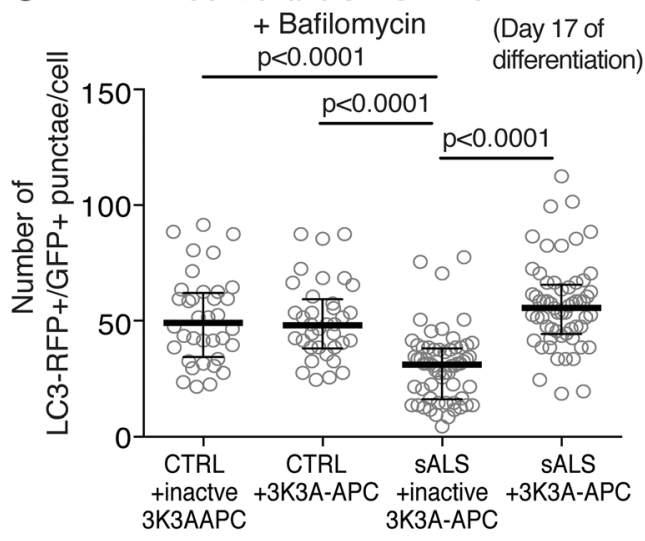

D (Day 17 of differentiation) (Day 17 of differentiation) C9-ALS2

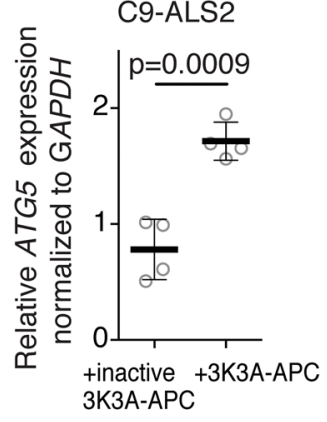
C9-ALS2

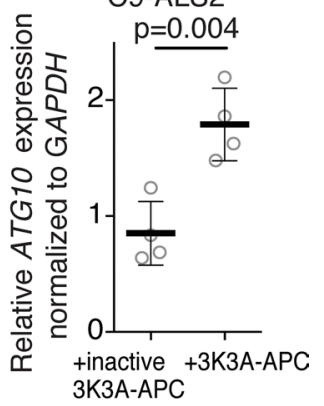

Figure 2. C9ORF72 and sporadic ALS iMNs share autophagosome formation abnormalities that are rescued by 3K3A-APC. (A) mRFP-GFP-LC3 fluorescence in control, C9-ALS, or sporadic ALS (sALS) iMNs treated with or without $50 \mathrm{nM}$ bafilomycin and $10 \mathrm{nM}$ inactive 3K3A-APC or 3K3A-APC. Scale bars: $5 \mu \mathrm{m}$. Solid and dotted lines outline the cell body and nucleus, respectively. Cell bodies were visualized with mRFP-GFP-LC3 fluorescence using a longer exposure and increased gain. (B and C) Number of RFP+CFP' vesicles per iMN in control, C9-ALS (B), or sporadic ALS (C) iMNs treated with 10 nM inactive 3K3A-APC or 3K3A-APC and 50 nM bafilomycin for 24 hours. iMNs from 3 controls, 3 C9-ALS (B), and 5 sporadic ALS (C) patients were quantified. $n=12$ iMNs per line per condition across 2 independent $i M N$ conversions were quantified. Each gray circle represents a single iMN. Median \pm interquartile range. Kruskal-Wallis testing. (D) qRT-PCR analysis of mRNA levels of ATC5 and ATC10 in C9-ALS iMNs treated with 10 nM inactive 3K3A-APC or 3K3A-APC 
for 3 days. $n=4$ independent iMN conversions and treatments per condition. Each gray circle represents a single RNA sample. Mean \pm SD. Two-tailed $t$ test, unpaired. (E) Number of LAMP2+ vesicles in control or C9-ALS iMNs treated with $10 \mathrm{nM}$ inactive 3K3A-APC or 3K3A-APC for 24 hours. iMNs from 2 controls and 2 C9-ALS patients were quantified. $n=21$ iMNs per line per condition across 2 independent iMN conversions were quantified. Each gray circle represents a single iMN. Mean \pm SEM. One-way ANOVA. (F) Number of LAMP2+ vesicles in control or sporadic ALS iMNs treated with $10 \mathrm{nM}$ inactive $3 K 3 A$ APC or 3K3A-APC for 24 hours. Each gray circle represents a single iMN. iMNs from 2 controls and 6 sporadic ALS patients were quantified. $n=33$ iMNs per line per condition across 2 independent $\mathrm{iMN}$ conversions were quantified. Median \pm interquartile range. Kruskal-Wallis testing. The day of differentiation stated on each panel indicates the day of differentiation on which the experimental treatment or time course was initiated.

in which 3 active-site lysine residues required for degrading factor Va and VIIIa are changed to alanine, resulting in a $90 \%$ reduction in anticoagulant activity but full retention of PAR1 activation ability (22). $3 \mathrm{~K} 3 \mathrm{~A}-\mathrm{APC}$ is well tolerated in mice under chronic dosing at levels that activate PAR1 within the central nervous system, and short-term peripheral administration is safe in monkeys (20, 23, 24). As a negative control for these studies, we used heat-inactivated 3K3A-APC.

$3 \mathrm{~K} 3 \mathrm{~A}-\mathrm{APC}$ potently rescued autophagosome formation in C9ORF72 ALS iMNs, as determined by its ability to increase the number of $\mathrm{GFP}^{+} \mathrm{mRFP}^{+}$vesicles in bafilomycin-treated iMNs (Figure 2, A and B [C9-ALS, inactive 3K3A-APC, +bafilomycin vs. C9-ALS, 3K3A-APC, +bafilomycin]; 3 controls and 3 C9ORF72 ALS patients). Interestingly, 3K3A-APC also increased autophagosome formation in sporadic ALS iMNs (Figure 2, A and C [sporadic ALS, inactive 3K3A-APC, +bafilomycin vs. sporadic ALS, 3K3AAPC, +bafilomycin]; 3 controls and 5 sporadic ALS patients, and Supplemental Figure 2B), indicating that is also capable of rescuing C9ORF72-independent autophagy impairments. Western blot analysis of C9ORF72 ALS motor neurons showed that 3K3A-APC increased the ratio of LC3-II to LC3-I in the presence of bafilomycin, verifying the results of the mRFP-GFP-LC3 assay (Supplemental Figure 2, C and D). Therefore, 3K3A-APC treatment rescues autophagosome formation in C9ORF72 and sporadic ALS iMNs.

To determine how 3K3A-APC might stimulate autophagosome formation, we performed RNA sequencing (RNA-Seq) on C9ORF72 ALS iMNs treated with 3K3A-APC or inactive 3K3A-APC for 3 days (2 C9ORF72 ALS patients). Consistent with our findings in the mRFP-GFP-LC3 and immunoblot assays, both Ingenuity Pathway Analysis (IPA) and KEGG pathway analysis revealed "autophagy" as one of the most significantly enriched gene sets (Supplemental Figure 2, E-H). RNA-Seq and quantitative realtime PCR (qRT-PCR) showed that 3K3A-APC significantly increased the expression of ATG5 and ATG10, which encode proteins with key roles in driving autophagosome formation (ref. 25, Figure 2D [qRT-PCR], Supplemental Figure 2I [RNA-Seq], and Supplemental Table 4). Thus, 3K3A-APC increases the expression of genes encoding autophagy-initiating factors in ALS iMNs.

Although mTOR inhibitors were ineffective at rescuing autophagosome formation in C9ORF72-deficient cells (16), we wondered whether this approach might be effective in sporadic ALS iMNs. Indeed, treatment with $10 \mu \mathrm{M}$ rapamycin significantly increased autophagosome formation in sporadic ALS iMNs (Supplemental Figure 2, J and K).

C9ORF72 ALS iMNs have fewer lysosomes than control iMNs (4), which could affect autophagic flux downstream of autophagosome formation. To determine if 3K3A-APC affects lysosome number in C9ORF72 ALS iMNs, we examined the number of LAMP2+ vesicles in iMNs (4). 3K3A-APC restored normal lysosome numbers in C9ORF72 ALS iMNs (Figure 2E; 2 controls and 2 C9ORF72 ALS patients, and Supplemental Figure 2, L and M). 3K3A-APC did not alter C9ORF72 protein (Supplemental Figure 2, N and O; 3 C9ORF72 ALS patients) or mRNA levels (Supplemental Figure 2P) in C9ORF72 ALS iMNs. Therefore, 3K3A-APC rescues lysosome numbers in C9ORF72 ALS iMNs without altering C9ORF72 levels.

iMNs from 3 of 6 sporadic lines also had significantly fewer LAMP2 ${ }^{+}$vesicles than control iMNs, and 3K3A-APC treatment rescued the number of lysosomes in these lines (Figure 2F and Supplemental Figure 2, $\mathrm{M}$ and $\mathrm{Q})$. Thus, iMNs from some, but not all sporadic ALS lines display low lysosome numbers. However, for those that do show low lysosome numbers, 3K3A-APC can rescue this phenotype.

Consistent with the notion that rescuing lysosome numbers in C9ORF72 ALS iMNs might enable autophagic flux even with increased autophagosome formation, the increase in the ratio of $\mathrm{GFP}^{+} \mathrm{m}$ $\mathrm{RFP}^{+}$vesicles to $\mathrm{GFP}^{-} \mathrm{mRFP}^{+}$vesicles upon $3 \mathrm{~K} 3 \mathrm{~A}-\mathrm{APC}$ treatment was modest compared with the overall increase in $\mathrm{GFP}^{+} \mathrm{mRFP}^{+}$vesicles in both C9ORF72 and sporadic ALS iMNs (Supplemental Figure 2, R and S; 3 C9ORF72 ALS patients and 5 sporadic ALS patients). Thus, 3K3A-APC treatment increases autophagy in C9ORF72 and sporadic ALS iMNs by rescuing both autophagosome formation and lysosome numbers. 
Rescue of autophagosome abnormalities by $3 \mathrm{~K} 3 \mathrm{~A}-\mathrm{APC}$ improves proteostasis. To determine if the effects of $3 \mathrm{~K} 3 \mathrm{~A}-\mathrm{APC}$ on stimulating autophagy in C9ORF72 and sporadic ALS iMNs were sufficient to lower DPR and cytoplasmic TDP-43 levels, we first measured levels of glycine-arginine [poly(GR)] and proline-arginine [poly(PR)] DPRs in C9ORF72 ALS iMNs with and without 3K3A-APC treatment. Treatment with $3 \mathrm{~K} 3 \mathrm{~A}-\mathrm{APC}$ for 6 days reduced the number of nuclear poly $(\mathrm{GR})^{+}$and poly $(\mathrm{PR})^{+}$punctae in $C 9 O R F 72 \mathrm{ALS}$ iMNs to control levels (Figure 3, A-C, and Supplemental Figure 3, A-E; 2 controls and 2 C9ORF72 ALS patients). Dot blot analysis for poly(GR) confirmed the decrease in iMNs after $3 \mathrm{~K} 3 \mathrm{~A}-\mathrm{APC}$ treatment (Figure 3, D and E). Moreover, 3K3A-APC reduced total nuclear and cytoplasmic levels of poly $(\mathrm{GR})^{+}$ and poly(PR) ${ }^{+}$punctae in C9ORF72 ALS iMNs (Supplemental Figure 3, F-H and J-L) and did not significantly alter the ratio of nuclear to cytoplasmic poly $(\mathrm{PR})^{+}$punctae, although it slightly lowered the ratio of nuclear to cytoplasmic poly $(\mathrm{GR})^{+}$punctae intensity (Supplemental Figure 3, I and M). These data indicate that 3K3A-APC treatment decreased both nuclear and cytoplasmic levels of DPRs. qRT-PCR using primers specific for C9ORF72 variants 1 and 3 (Supplemental Table 3) showed that 3K3A-APC did not reduce RNA levels of the repeat expansion (Supplemental Figure $3 \mathrm{~N}$ ), excluding the possibility that the reduced DPR levels were caused by transcriptional repression. 3K3A-APC also did not alter the expression of genes associated with the integrated stress response (Supplemental Figure 3O), indicating that its ability to lower DPR levels was independent of this pathway.

Since 3K3A-APC treatment lowered DPR levels, we wondered if this effect was significant enough to prevent DPR-induced neurodegeneration. To test this, we measured the ability of $3 \mathrm{~K} 3 \mathrm{~A}-\mathrm{APC}$ to rescue the degeneration caused by the overexpression of a GR(50)-GFP fusion protein in control iMNs. 3K3A-APC significantly reduced the total intensity of GR(50)-GFP and GR(50)-GFP punctae size, indicating that it reduced GR(50)-GFP levels (Supplemental Figure 3, P and Q). Moreover, 3K3A-APC increased the survival of GR(50)-GFP-expressing iMNs, indicating that it prevents DPR-induced neurodegeneration (Figure 3F).

Because autophagy induction can enhance TDP-43 turnover in motor neurons (26), we wondered whether 3K3A-APC treatment could prevent the cytoplasmic accumulation of TDP-43 in ALS iMNs. $3 \mathrm{~K} 3 \mathrm{~A}-\mathrm{APC}$ treatment for 6 days significantly increased the nuclear-to-cytoplasmic ratio of TDP-43 in C9ORF72 and sporadic ALS iMNs to control levels (Figure 3, G-J; 2 controls, 2 C9ORF72 ALS, and 6 sporadic ALS patients). Thus, 3K3A-APC treatment can rescue autophagosome formation, lower DPR levels in C9ORF72 ALS iMNs and rescue DPR-mediated neurotoxicity, and reverse the cytosolic accumulation of TDP-43 in C9ORF72 and sporadic ALS patient iMNs.

C9ORF72 and sporadic ALS iMNs have elevated glutamate receptor levels that are normalized by $3 K 3 A-A P C$. Studies in patients and animal and cell models have linked neuronal hyperexcitability to ALS (6, 27-32), but it remains unclear if there are common cell-autonomous mechanisms that drive hyperexcitability in different ALS patients. We previously found that reduced C9ORF72 levels lead to elevated surface-bound levels of glutamate receptors in C9ORF72 ALS iMNs, C9ORF72-deficient mice, and C9ORF72 patients, and that these differences were not caused by differential gene expression (4). We also showed that the elevated glutamate receptor levels increased sensitivity to glutamate-induced excitotoxicity in vitro and in vivo (4).

To determine if increased glutamate receptor levels might be a conserved mechanism that drives hyperexcitability in multiple forms of ALS, we examined levels of the NR1 NMDA receptor subunit in neurites of control, C9ORF72 ALS, and sporadic ALS iMNs by immunostaining. Similarly to our previous study, C9ORF72 iMNs possessed significantly more $\mathrm{NR}^{+}$punctae on neurites than control iMNs (Figure 4, A and B; 2 controls and 2 C9ORF72 ALS patients, and ref. 4). Colabeling with a MAP2-specific antibody verified that the $\mathrm{NR} 1^{+}$punctae that were increased in abundance were localized on dendrites (Supplemental Figure 4, A and B). Interestingly, sporadic ALS iMNs also displayed more $\mathrm{NR}^{+}$punctae on neurites than control iMNs (Figure 4, A and C, and Supplemental Figure 4, C-H; 2 controls and 6 sporadic ALS patients). Calcium imaging confirmed that C9ORF72 and sporadic ALS iMNs experienced more calcium transients than controls in response to glutamate, indicating that they may be more sensitive to excitotoxicity (Figure 4, D and E; 3 controls, 3 C9ORF72 ALS patients, 1 sporadic ALS patient). Thus, both C9ORF72 and sporadic ALS iMNs display increased NR1 levels, which could reflect a shared mechanism of increased susceptibility to excitotoxicity.

Because glutamate receptor homeostasis is maintained in part through vesicle trafficking $(33,34)$ and we had observed that $3 \mathrm{~K} 3 \mathrm{~A}-\mathrm{APC}$ exerted potent effects on autophagosomal and lysosomal pathways in iMNs, we determined if 3K3A-APC could normalize glutamate receptor levels in ALS patient-derived motor neurons. Indeed, 3K3A-APC reduced the number of $\mathrm{NR}^{+}$punctae on C9ORF72 and sporadic ALS iMN neurites to 
A
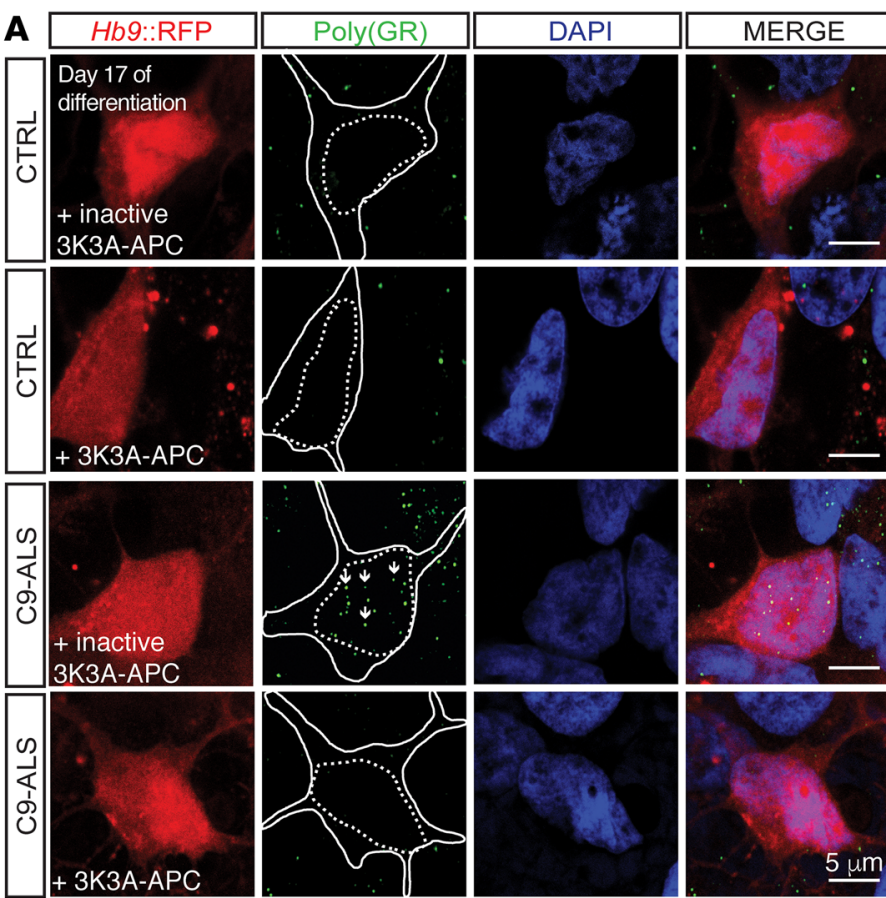

B ( Day 17 of differentiation
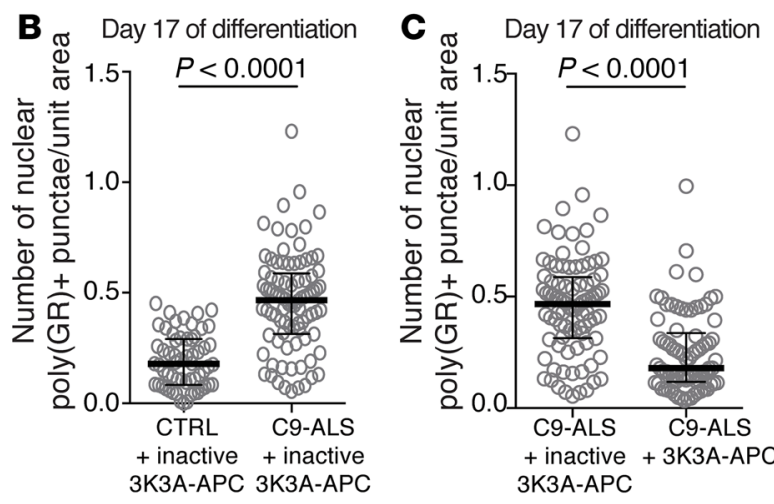

D

C9-ALS + inactive $3 \mathrm{~K} 3 \mathrm{~A}-\mathrm{APC}$

C9-ALS +

Day 17 of $\overline{\text { Rep1 Rep2 Rep3 Rep1 Rep2 Rep3 }}$

poly(GR)

TUJ1

E

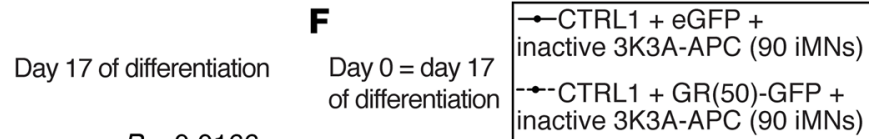

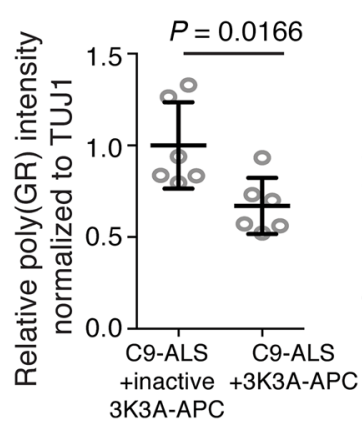

(2 patients)

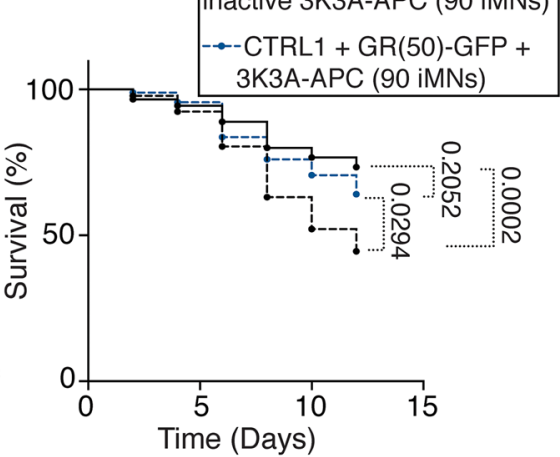

$5 \underline{\mu \mathrm{m}}$
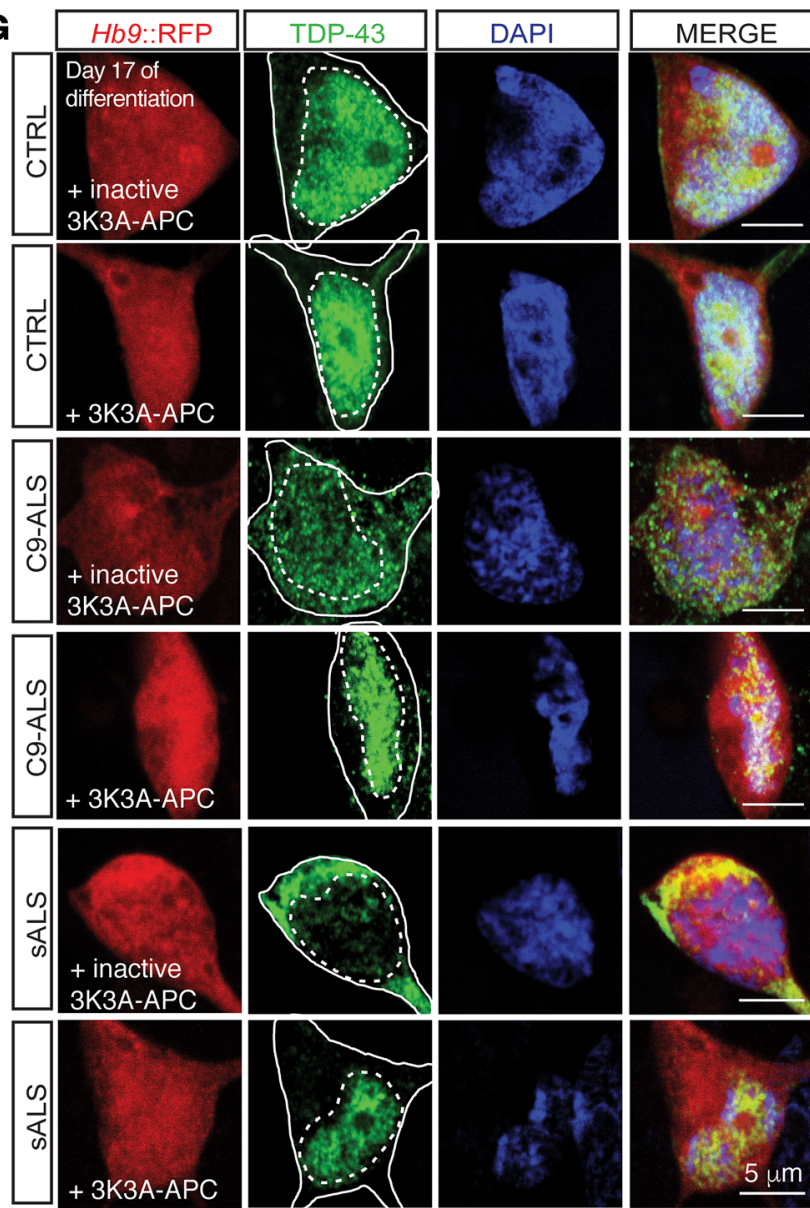

H

Day 17 of differentiation
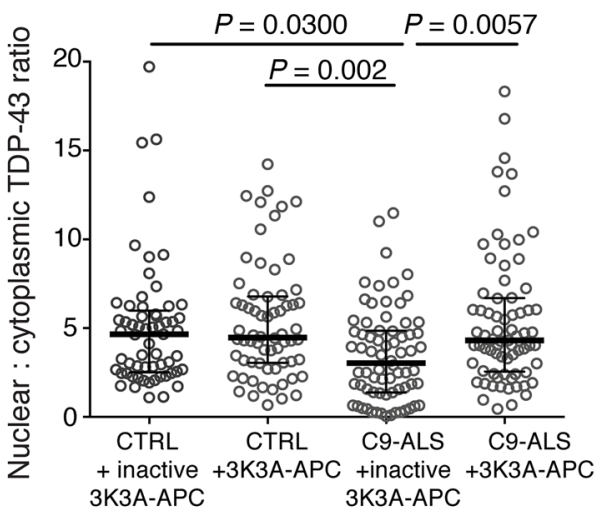

I
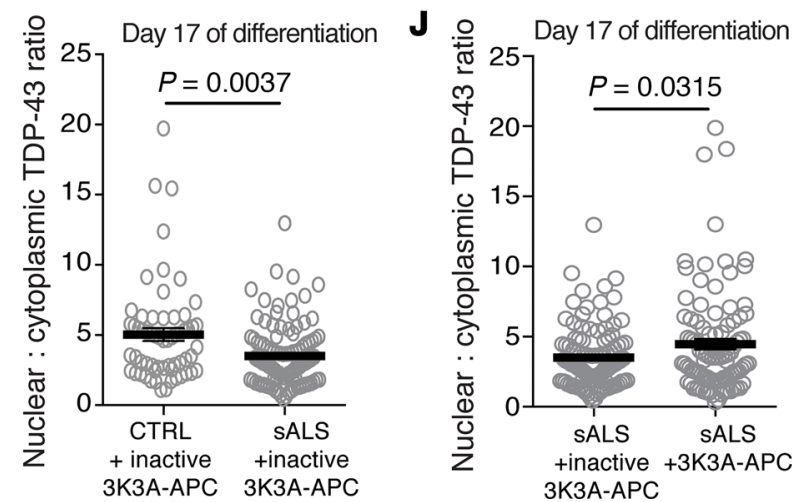
Figure 3. Rescue of autophagosome formation by 3K3A-APC improves proteostasis. (A-C) Immunostaining (A) and quantification (B and C) to determine endogenous poly(GR)+ punctae in control or C9-ALS iMNs with $10 \mathrm{nM}$ inactive 3K3A-APC or 3K3A-APC treatment for 6 days. Quantified values represent the average number of nuclear poly(GR)+ punctae in $n=30 \mathrm{iMNs}$ (controls) or 40 to $44 \mathrm{iMNs}$ (C9-ALS) per line per condition from 2 control or 2 C9-ALS patient lines. For each line, iMNs were quantified from 2 independent iMN conversions per line per condition. Median \pm interquartile range. Each gray circle represents the number of poly(GR)+ punctae/unit area in a single iMN. Mann-Whitney testing. Solid and dotted lines in $\mathbf{A}$ outline the cell body and nucleus, respectively. Scale bars: $5 \mu \mathrm{m}$. (D and E) Dot blot (D) and quantification (E) of poly(CR)+ levels in iMNs from 2 C9-ALS patient lines with $10 \mathrm{nM}$ inactive 3K3A-APC or 3K3A-APC treatment for 6 days. Each gray circle represents 1 dot blot sample. Mean \pm SD. $n=3$ independent iMN conversions per line per condition. One-way ANOVA with Tukey's correction across all comparisons. (F) Survival of control iMNs without excess glutamate with overexpression of eGFP or GR(50)-eGFP and $10 \mathrm{nM}$ inactive or active 3K3A-APC. $n=90$ iMNs per condition, iMNs quantified from 3 biologically independent iMN conversions. Two-sided log-rank test, corrected for multiple comparisons, statistical significance was calculated using the entire survival time course. $n=90$ iMNs per condition. (G-J) Immunofluorescence analysis of total TDP-43 (G) and quantification of the ratio of nuclear to cytoplasmic TDP-43 in control, C9-ALS (H), or sporadic ALS iMNs (I and J). Ratio of nuclear to cytoplasmic TDP-43 in individual C9-ALS iMNs treated with $10 \mathrm{nM}$ inactive 3K3A-APC or 3K3A-APC for 6 days. iMNs from 2 controls, 2 C9-ALS, and 4 sporadic ALS patients were quantified. $n=30$ iMNs per line (control and C9-ALS) per condition or $n=26$ iMNs (I), $30 \mathrm{iMNs}$ (J) (inactive 3K3A-APC), or $n=35 \mathrm{iMNs}$ (J) (3K3A-APC) (sporadic ALS) per condition per line from 2 biologically independent iMN conversions were quantified. Each gray circle represents a single iMN. For $\mathbf{H}$, median \pm interquartile range. Kruskal-Wallis testing. For I and J, mean \pm SEM. Unpaired $t$ test with Welch's correction. Scale bars: $5 \mu \mathrm{m}$. Dotted lines outline the nucleus and cell body. The day of differentiation stated on each panel indicates the day of differentiation on which the experimental treatment or time course was initiated.

control iMN levels (Figure 4, A-C, and Supplemental Figure 4, A and B; 2 controls, 2 C9ORF72 ALS, and 6 sporadic ALS patients). Using surface protein biotinylation, we were able to purify surface-bound proteins from iMNs (Figure 4, F-I, and Supplemental Figure 4I). Immunoblottting confirmed that 3K3A-APC reduced membrane-bound NR1 levels on C9ORF72 and sporadic ALS iMNs (Figure 4, F-I, and Supplemental Figure 4, J-M), but not control iMNs (Supplemental Figure 4, N-Q). 3K3A-APC treatment did not alter total NR1 levels, but specifically reduced the amount of surface-bound NR1 on ALS iMNs (Supplemental Figure 4, J-M and R-W). 3K3A-APC also did not alter total NR1 levels in control iMNs (Supplemental Figure 4, X-Z). Thus, 3K3A-APC normalizes NR1 levels on C9ORF72 and sporadic ALS iMNs.

$3 K 3 A-A P C$ rescues the survival of C9ORF72 and sporadic ALS iMNs in a PAR1-dependent manner. We next determined if 3K3A-APC's ability to rescue autophagosome production, increase lysosomal numbers, lower levels of DPRs and mislocalized TDP-43, and lower glutamate receptor levels could rescue the degeneration of ALS iMNs. We first tested the ability of the iMN survival assay to replicate the neuroprotective effects previously shown for activated protein C in SOD1G93A mice (20). iMNs derived from an ALS patient carrying an SOD1A4V mutation degenerated significantly faster than control iMNs (Figure 5A and Supplemental Figure 5A; 3 controls and 1 SOD1A4V ALS patient) and 3K3A-APC rescued their survival (Figure 5A and Supplemental Figure 5A; 1 SOD1A4V ALS patient). Thus, the iMN model mimics disease biology observed in vivo.

3K3A-APC potently rescued the survival of iMNs from all 3 C9ORF72 ALS lines in a dose-dependent manner (Figure 5, B and C, and Supplemental Figure 5, B and C; 3 C9ORF72 ALS patients). In contrast, 3K3A-APC did not improve the survival of control iMNs (Figure 5D and Supplemental Figure 5D; 3 controls). Activated protein $C$ can mediate neuroprotective effects by activating PAR1 receptor signaling in neurons $(20,35)$ and RNA-Seq data confirmed that iMNs express high levels of the F2R gene, which encodes PAR1 (Supplemental Figure 5E). Thus, we measured C9ORF72 ALS iMN survival in the presence or absence of PAR1, PAR2, or PAR3 antisense oligonucleotides (ASOs) or small-molecule antagonists. Chemical inhibition of PAR1 or PAR2 alone did not affect C9ORF72 ALS iMN survival (Supplemental Figure 5, F and G; 2 C9ORF72 ALS patients). However, PAR1 antagonist treatment blocked the ability of 3K3A-APC to rescue C9ORF72 ALS iMN survival (Figure 5E and Supplemental Figure 5H; 2 C9ORF72 ALS patients), while a PAR2 antagonist did not (Figure 5F and Supplemental Figure 5I; 2 C9ORF72 ALS patients). Consistent with these findings, ASO-mediated suppression of PAR 1 significantly reduced the neuroprotective effect of 3K3A-APC on C9ORF72 ALS iMN survival (Figure 5G and Supplemental Figure 5, J and K; 2 C9ORF72 ALS patients, and Supplemental Table 3). In contrast, ASO-mediated suppression of PAR 2 or $P A R 3$ had no effect on the ability of $3 \mathrm{~K} 3 \mathrm{~A}$ APC to rescue C9ORF72 ALS iMN survival (Supplemental Figure 5, L-Q; 2 C9ORF72 ALS patients). Therefore, 3K3A-APC rescues C9ORF72 ALS iMN survival through activation of PAR1.

3K3A-APC also rescued the survival of iMNs from all 6 sporadic ALS lines (Figure 5H and Supplemental Figure 6, A-F; 6 sporadic ALS patients). When we tested iMNs from separate iPSC clones of the sporadic patient lines, 3K3A-APC also significantly improved their survival, indicating that the rescue effects were not specific to iMNs derived from certain iPSC clones (Supplemental Figure 6, G-J; 3 sporadic ALS patients). 
A

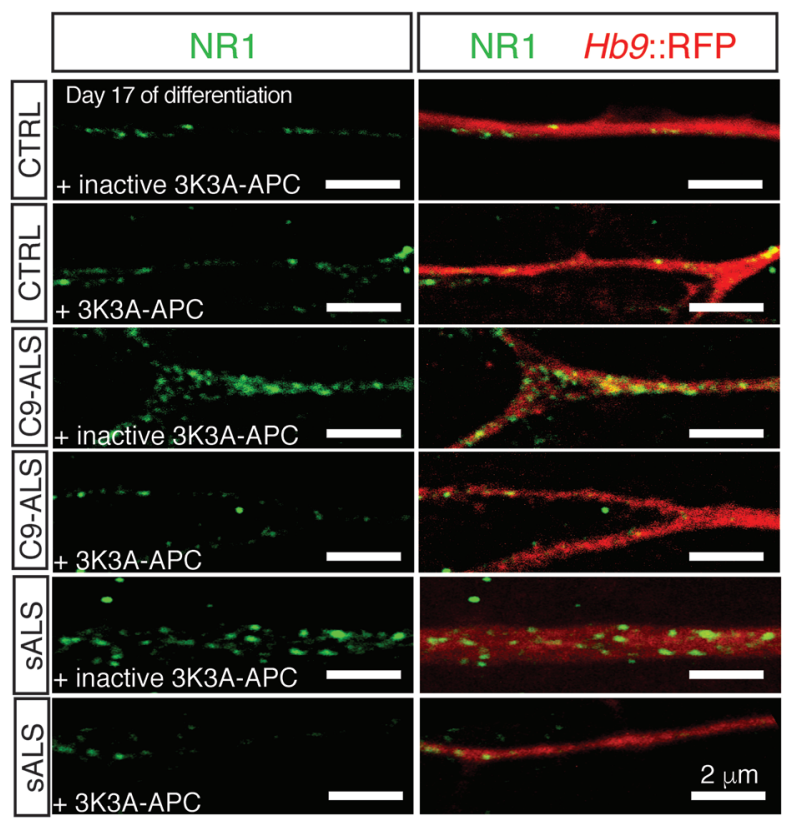

B

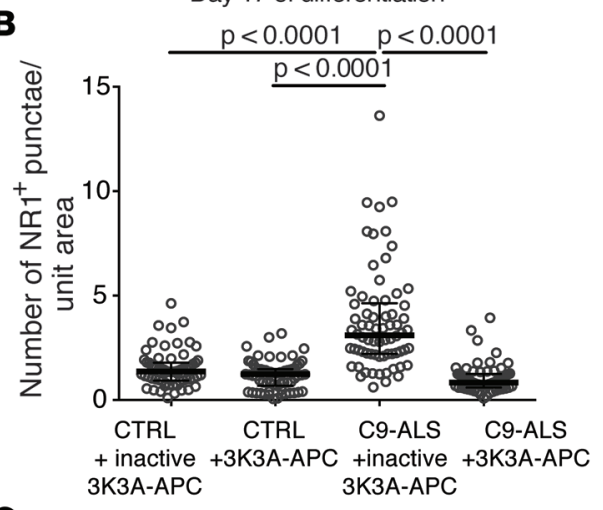

C

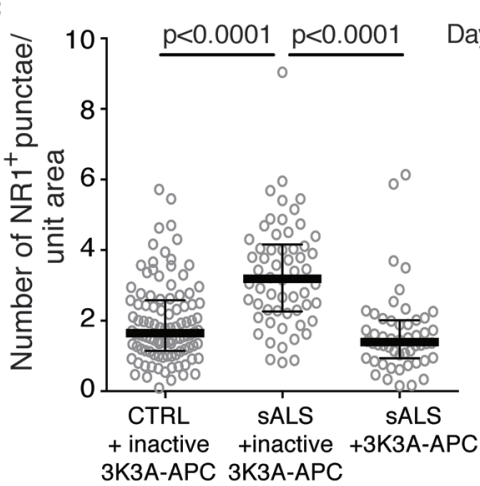

D

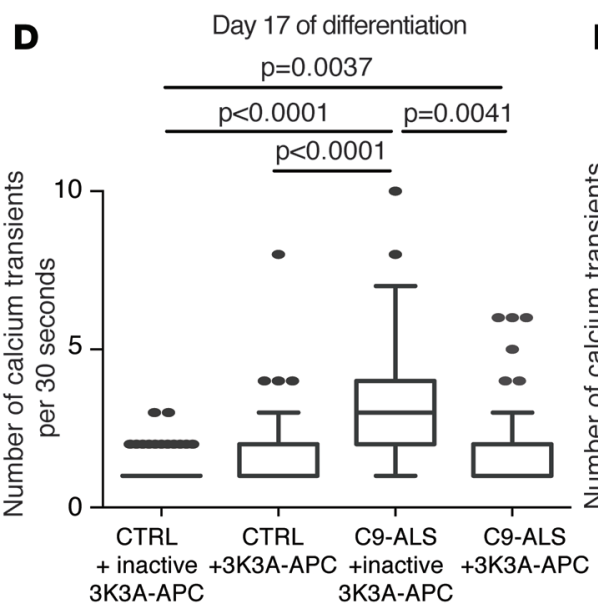

E

Day 17 of differentiation

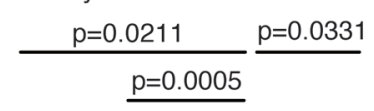

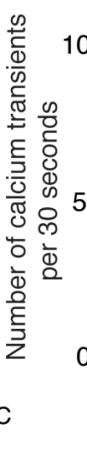

$p=0.0005$

G

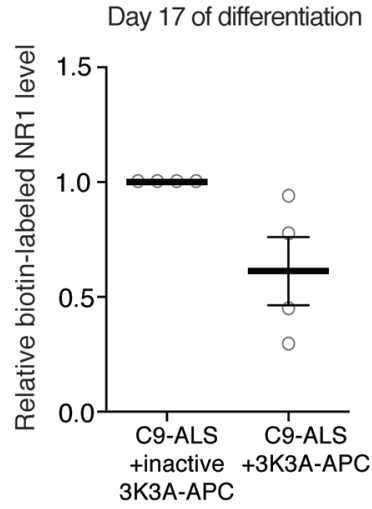

H

MW Inactive

(KDa) 3K3A-APC 3K3A-APC

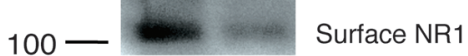

$70 \longrightarrow$ Surface TF Receptor

$70--$ Total TF Receptor

55 - Total TUJ1
+ inactive +3K3A-APC +inactive +3K3A-APC उK3A-APC
$\mathbf{F}$

C9-ALS iMNs Day 17 of differentiation

MW Inactive

(kDa) 3K3A-APC 3КЗА-APC

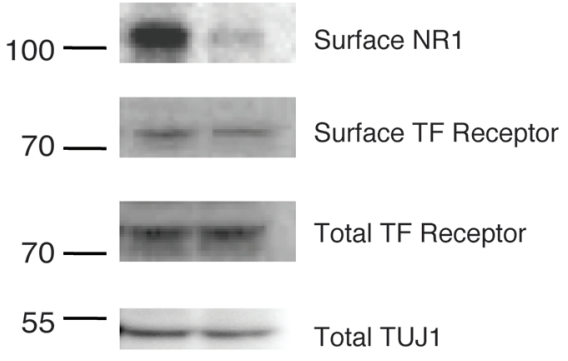

Total TUJ 
Figure 4. C9ORF72 and sporadic ALS iMNs have elevated glutamate receptor levels that are normalized by 3K3A-APC. (A) Immunofluorescence images showing NR1 $1^{+}$punctae on neurites of iMNs treated with $10 \mathrm{nM}$ inactive 3K3A-APC or 3K3A-APC for 6 days. Scale bar: $2 \mu \mathrm{m}$. This experiment was repeated 3 times with similar results. (B and C) NR1+ punctae per unit area in control, C9-ALS (B), or sporadic ALS (C) iMNs. Each gray circle represents the number of NR1 $1^{+}$punctae per area unit on a single neurite (1 neurite quantified per iMN). $n=33$ (controls and C9-ALS) or 13 (sporadic) iMNs quantified per line per condition from 2 biologically independent iMN conversions of 2 CTRL, 2 C9-ALS, or 6 sporadic ALS lines. Median \pm interquartile range. Kruskal-Wallis testing. (D) Number of calcium transients per 30 seconds in control or C9-ALS iMNs treated with $10 \mathrm{nM}$ inactive 3K3A-APC or 3K3A-APC. $n=21 \mathrm{iMNs}$ per line per condition from 3 biologically independent iMN conversions of 3 CTRL and 3 C9-ALS lines. For the C9-ALS plus 3K3A-APC condition, $n=19$ iMNs per line. Median \pm interquartile range. Kruskal-Wallis testing. (E) Number of calcium transients per 30 seconds in control or sporadic ALS iMNs treated with inactive 3K3A-APC or 3K3A-APC. $n=20$ iMNs per line per condition from 3 biologically independent $\mathrm{MNN}$ conversions of 3 CTRL and $1 \mathrm{sporadic}$ line. Median \pm interquartile range. Kruskal-Wallis testing. (F) Immunoblotting of surface NR1 after surface protein biotinylation in C9-ALS iMNs generated with NGN2, ISL1, and $L H X 3$ and treated with $10 \mathrm{nM}$ inactive 3K3A-APC or 3K3A-APC for 6 days. (C) Quantification of NR1 immunoblotting from F. $n=4$ biologically independent iMN conversions. Each gray circle represents an individual sample. The ratio of surface to total transferrin receptor was used to normalize for the membrane protein extraction efficiency and TUJ1 was used to normalize for neuron number. (H) Immunoblotting of surface NR1 after surface protein biotinylation in sporadic ALS iMNs (1 patient) generated with NCN2, ISL1, and $L H X 3$ and treated with 10 nM inactive 3K3A-APC or 3K3A-APC for 6 days. The full blot for total TUJ1 is shown. (I) Quantification of NR1 immunoblotting from H. $n=4$ biologically independent iMN conversions. Each gray circle represents an individual sample. The ratio of surface to total transferrin receptor was used to normalize for the membrane protein extraction efficiency and TUJ1 was used to normalize for neuron number. The day of differentiation stated on each panel indicates the day of differentiation on which the experimental treatment or time course was initiated. TF, transferrin.

Reinforcing the notion that activating autophagosome formation in ALS iMNs could increase survival, rapamycin treatment significantly improved the survival of iMNs from 4 of 5 sporadic ALS lines tested (Supplemental Figure 7, A-J; 6 sporadic ALS patients). In contrast, rapamycin slightly decreased the survival of control iMNs (Supplemental Figure 7, K and L). These results suggest that rescuing autophagosome formation in sporadic ALS iMNs can increase their survival.

Treatment with the small-molecule PAR1 antagonist alone did not affect the survival of sporadic ALS iMNs (Supplemental Figure 6, K-P; 6 sporadic ALS patients), but the PAR1 antagonist blocked the ability of 3K3A-APC to rescue sporadic ALS iMN survival (Figure 5I and Supplemental Figure 6, Q-V; 6 sporadic ALS patients). Thus, 3K3A-APC can rescue C9ORF72 and sporadic ALS iMN survival through activation of PAR1.

$3 K 3 A-A P C$ rescues C9ORF72 ALS proteostasis and glutamate receptor phenotypes in vivo. We next wondered if $3 \mathrm{~K} 3 \mathrm{~A}-\mathrm{APC}$ could lower DPR levels in vivo. Baloh and colleagues previously generated C9-BAC mice that harbor a human C9ORF72 gene containing 100-1000 GGGGCC repeats and produce DPRs that aggregate in neurons (36). Forty-eight hours after direct injection into the hippocampus, 3K3A-APC-injected hippocampi showed a significant reduction in $\operatorname{poly}(\mathrm{GR})^{+}, \operatorname{poly}(\mathrm{PR})^{+}$, and poly $(\mathrm{GP})^{+}$punctae (Figure $\left.6, \mathrm{~A}-\mathrm{D}\right)$. Thus, in vivo, 3K3A-APC can reduce levels of both nuclear and cytoplasmically localized DPRs, as well as sense- and antisense-transcript-derived DPRs.

To determine if 3K3A-APC can normalize glutamate receptor levels on neurons in vivo, we examined its ability to lower NR1 levels and NMDA-induced excitotoxicity in C9orf $72^{+/-}$mice. We previously showed that hippocampal, cortical, and spinal cord neurons of $C 90 r f 72^{+/-}$mice have increased NR1 levels compared with C9orf $72^{+/+}$mice (4). We previously developed an acute, in vivo NMDA-induced excitotoxicity assay in the hippocampus (Figure $6 \mathrm{E}$ ), and verified using cresyl violet staining as well as Fluoro-Jade B-positive dead neuron counting in sham control mice that the operation procedure itself does not result in significant injury (4). Similarly to iMN cultures, reduced C9ORF72 levels in C9orf72 ${ }^{+/-}$and C9orf $72^{-1-}$ mice cause hippocampal neurons to undergo greater NMDA-induced excitotoxicity than in C9orf $72^{+/+}$mice, resulting in a significantly larger loss of neurons in C9ORF72-deficient mice that could be detected by a reduction in cresyl violet staining (Figure 6, F and G, and ref. 4). A single injection of $3 \mathrm{~K} 3 \mathrm{~A}-\mathrm{APC}$ into the hippocampus of $\mathrm{C} 9 \mathrm{orf} 2^{+/-}$mice significantly lowered NR1 levels after 48 hours (Figure $6, \mathrm{H}$ and I) ( $n=3$ mice). Moreover, when coinjected with NMDA, 3K3A-APC significantly reduced the amount of NMDA-induced hippocampal neurodegeneration in $C 90 r f 2^{+/-}$mice (Figure $6, \mathrm{~F}$ and $\mathrm{G}$ ). Thus, in vivo, 3K3A-APC improves proteostasis and normalizes glutamate receptors, thereby rescuing critical C9ORF72 ALS/FTD gain- and loss-of-function disease processes, respectively.

\section{Discussion}

Our data indicate that motor neurons derived from C9ORF72 and at least a subset of sporadic ALS patients share similar defects in autophagosome formation and glutamate receptor accumulation. Importantly, PAR1 activation by $3 \mathrm{~K} 3 \mathrm{~A}-\mathrm{APC}$ can reverse these C9ORF72 and sporadic ALS disease processes in vitro and in vivo (Figure 7). We draw several key conclusions from our findings. 
A

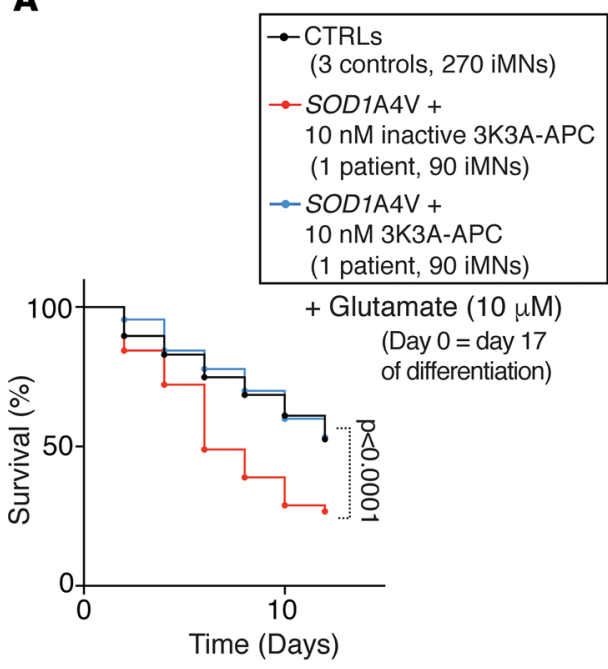

B

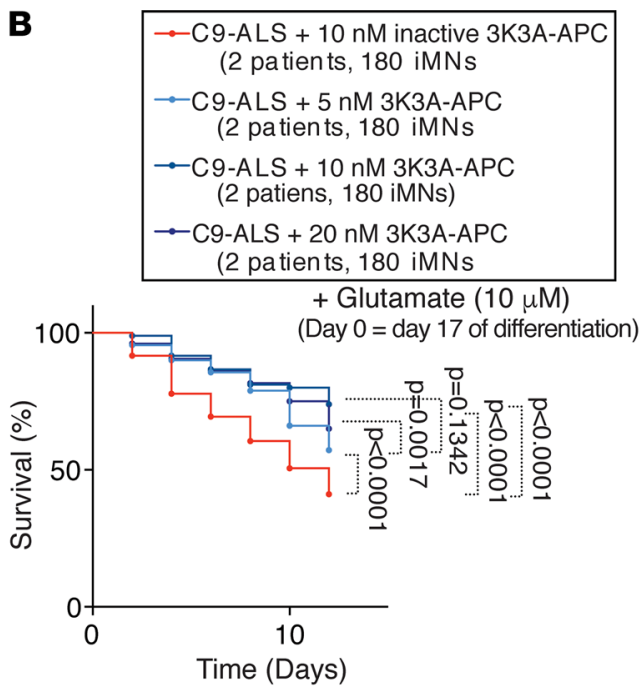

C

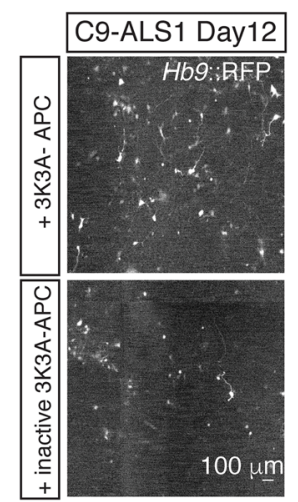

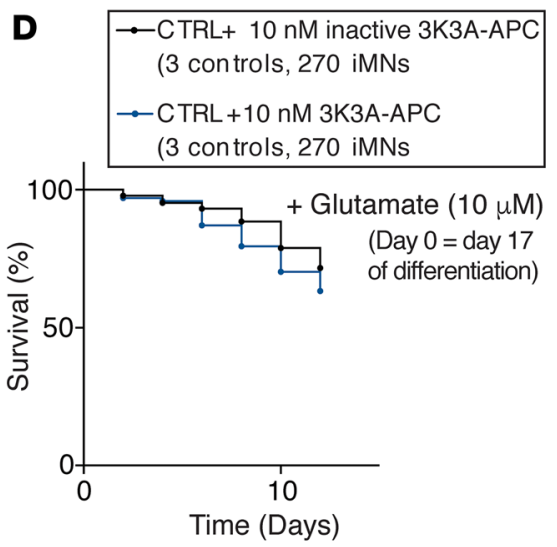

G

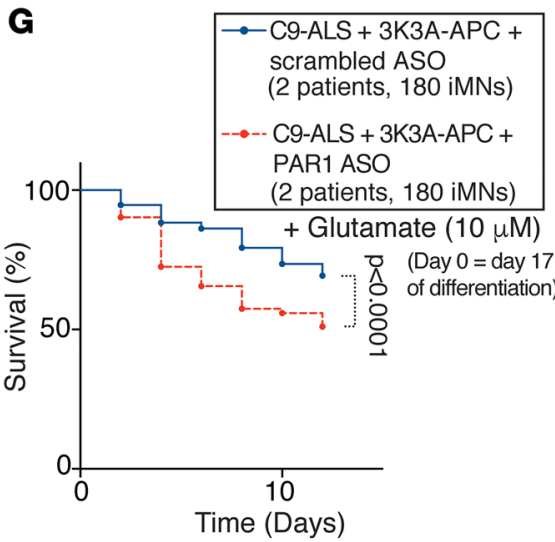

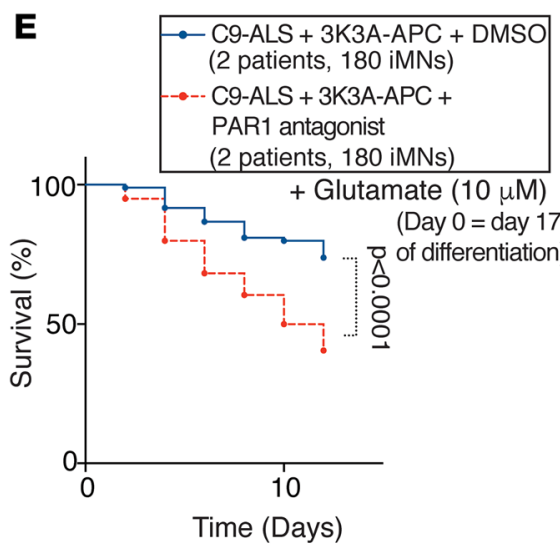

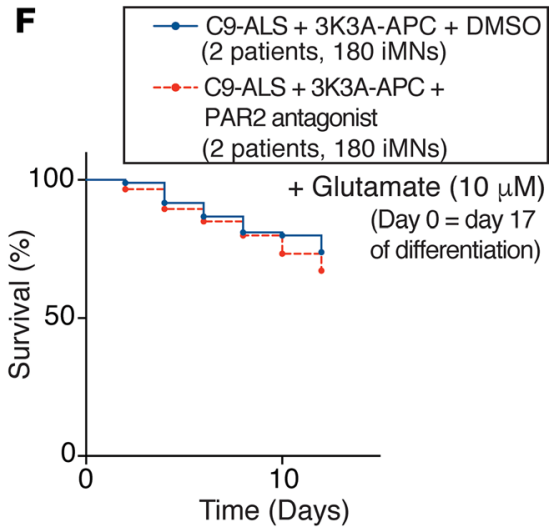

H

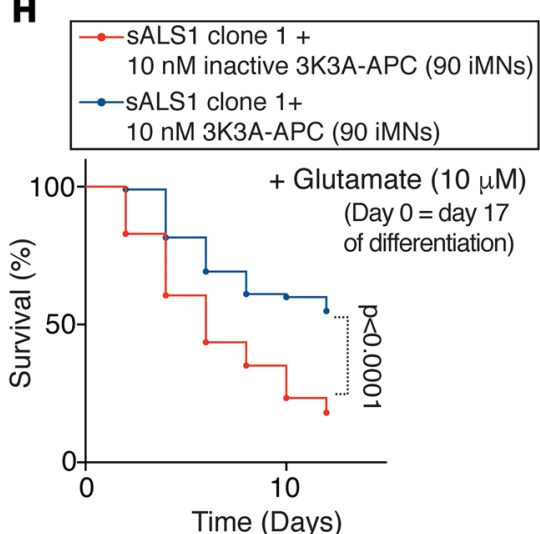

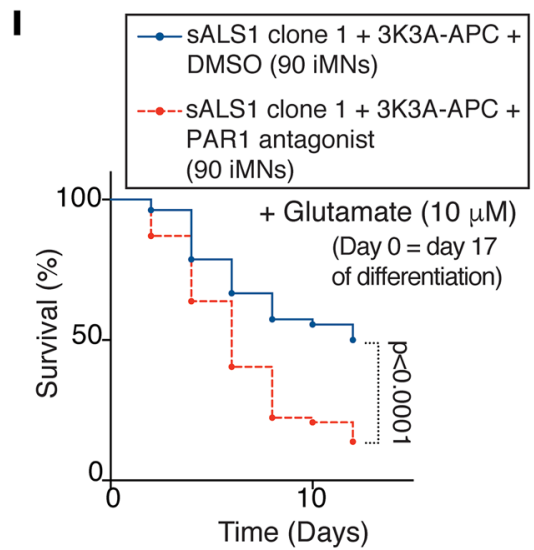

Figure 5. 3K3A-APC rescues the survival of C9ORF72 and sporadic ALS iMNs in a PAR1-dependent manner. (A) Survival of iMNs from 3 control or 1 SOD1A4V ALS patient line in excess glutamate with $10 \mathrm{nM}$ inactive 3K3A-APC or 3K3A-APC. $n=90$ iMNs per line per condition, iMNs from all control lines shown in aggregate for clarity. iMNs quantified from 3 biologically independent iMN conversions per line. (B) Survival of iMNs from 2 C9-ALS lines in excess glutamate with inactive 3K3A-APC or different concentrations of 3K3A-APC. $n=90$ iMNs per line per condition, iMNs from both lines shown in aggregate for clarity. iMNs quantified from 3 biologically independent iMN conversions per line. (C) C9-ALS iMNs on day 12 of survival in excess glutamate with inactive 3 K3A-APC or 3K3A-APC treatment. This experiment was repeated 3 times with similar results. Scale bar: $100 \mu \mathrm{m}$. (D) Survival of control iMNs in excess glutamate with $10 \mathrm{nM}$ inactive 3 K3A-APC or 3K3A-APC, $n=90$ iMNs per line per condition for 3 control and 3 C9-ALS lines, iMNs quantified from 3 biologically independent iMN conversions per line. (E-G) Survival of iMNs from 2 C9-ALS lines in excess glutamate with 3K3A-APC with or without $3 \mu$ M PAR1 antagonist treatment (E) or PAR2 antagonist treatment $(\mathbf{F}) . n=90$ iMNs per line per condition, iMNs from both lines shown in aggregate for clarity. iMNs quantified from 3 biologically independent iMN conversions per line. Survival of iMNs from 2 C9-ALS lines in excess glutamate with 3K3A-APC with or without $9 \mu$ M PAR1 ASO treatment (G). $n=90$ iMNs per line per condition, iMNs from both lines shown in aggregate for clarity. iMNs quantified from 3 biologically independent iMN conversions per line. Each trace includes neurons from 2 donors with the specified genotype. All iMN survival experiments were analyzed by 2-sided log-rank test and corrected for multiple 
comparisons if applicable. Statistical significance was calculated using the entire survival time course. (H and I) Survival of iMNs from sporadic ALS (sALS) clone 1 in excess glutamate with $10 \mathrm{nM}$ inactive 3K3A-APC or 3K3A-APC (H), or with 3K3A-APC and DMSO or a PAR1 antagonist (I). $n=90$ iMNs per condition. iMNs quantified from 3 biologically independent iMN conversions. For all iMN survival experiments, significance was measure by 2-sided log-rank test using the entire survival time course. The day of differentiation stated on each panel indicates the day of differentiation on which the experimental treatment or time course was initiated.

First, motor neurons derived from 6 sporadic ALS patients selected without bias toward certain genetic mutations or clinical symptoms display defects in autophagosome formation and accumulate NMDA receptors on their neurites. Both C9ORF72 and sporadic ALS iMNs exhibit these phenotypes, revealing an important area of mechanistic convergence between C9ORF72 ALS and at least some forms of sporadic ALS.

A previous study found that in postmortem tissue from sporadic ALS patients, motor neurons may have had lower levels of NMDA receptors (37). However, this may be because the motor neurons containing the highest amounts of NMDA receptors were most vulnerable to degeneration. Alternatively, the patients examined postmortem in this study may have differed from the patients in our cohort. This highlights the importance of expanding the number of sporadic ALS patient iPSC lines.

There are at least 3 potential reasons for the shared abnormalities in autophagosome formation and glutamate receptor levels we have observed. First, some sporadic ALS patients may harbor genetic variants that directly impair autophagosome formation. If so, this could induce the autophagosome formation defects that in turn could cause excess glutamate receptor accumulation. Alternatively, genetic variants affecting other aspects of proteostasis could lead to the accumulation of unfolded proteins, which might impair autophagosome formation through some unknown mechanism. The accumulation of unfolded protein could also impair overall protein turnover and lead to glutamate receptor accumulation. Third, the sporadic ALS patients could harbor genetic variants that affect vesicular signaling molecules, such as phosphatidylinositols, that regulate both endocytosis of surface proteins and autophagosome formation (38). It will also be important to determine how conserved these neuronal phenotypes are in other neurodegenerative diseases. One study has clearly shown that a Parkinson's disease mutation in synaptojanin impairs autophagosome formation in iPSC neurons (38).

A second key conclusion is that a single therapeutic approach that rescues the autophagosome and glutamate receptor abnormalities we discovered can slow the degeneration of ALS patient motor neurons. Importantly, we show that this approach is effective for both C9ORF72 and sporadic ALS patients.

A third conclusion from our findings is that a single therapeutic approach can rescue both gain- and lossof-function C9ORF72 disease processes. 3K3A-APC treatment reduces levels of NMDA receptors on iMNs and hippocampal neurons in vivo and rescues NMDA-induced excitotoxicity in C9orf72-deficient mice. Our observation that $3 \mathrm{~K} 3 \mathrm{~A}-\mathrm{APC}$ stimulates autophagosome formation indicates that $3 \mathrm{~K} 3 \mathrm{~A}-\mathrm{APC}$ can also rescue this aspect of the loss of C9ORF72 function. 3K3A-APC significantly lowers DPR levels in C9ORF72 ALS iMNs and C9ORF72 BAC transgenic mice, thereby mitigating this gain-of-function disease process.

A fourth implication of our study is that a single therapeutic agent can reduce levels of DPRs produced from both the sense and antisense C9ORF72 transcripts as well as nuclear and cytoplasmic DPRs, suggesting that it is effective against all DPR species.

Our findings provide preclinical evidence suggesting that 3K3A-APC and PAR1 activation could rescue neurodegeneration in C9ORF72 and sporadic ALS cases. In complex diseases such as ALS, the identification of therapeutic strategies that are efficacious across a wide range of cases is critical for clinical success. We have developed tools, including C9ORF72 and sporadic ALS iMN disease models, that enable the identification of these rare, but critical therapeutic strategies.

\section{Methods}

iPSC reprogramming. Human lymphocytes from healthy subjects and ALS patients were obtained from the NINDS Biorepository at the Coriell Institute and reprogrammed into iPSCs as previously described (39).

Molecular cloning and viral production. The cDNA for each iMN transcription factor or the mRFP-GFPLC3 construct was subcloned into the pMXs retroviral expression vector (4). The Hb9::RFP lentiviral vector was previously purchased from Addgene (ID: 37081). Viruses were produced as follows. HEK $293 \mathrm{~T}$ cells (ATCC, CRL-11268) were transfected at $80 \%$ to $90 \%$ confluence with viral vectors containing genes of interest and viral packaging plasmids (PIK-MLV-gp and pHDM for retrovirus, pPAX2 and VSVG for lentivirus) using polyethylenimine (Sigma-Aldrich). HEK 293T were tested for mycoplasma before, during, 
A Inject at Anteroposterior -2.0, Mediolateral +1.5, Dorsoventral -1.8,

$.2 \mu \mathrm{g} 3 \mathrm{~K} 3 \mathrm{~A}-\mathrm{APC}$ or inactive $3 \mathrm{~K} 3 \mathrm{~A}-\mathrm{APC}$ in a $.3 \mu \mathrm{l}$ volume

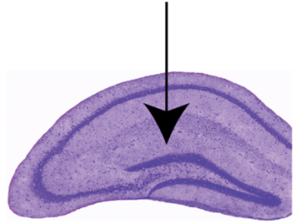

48 hours later

Analyze dipeptide repeat protein levels

$\mathbf{B}$

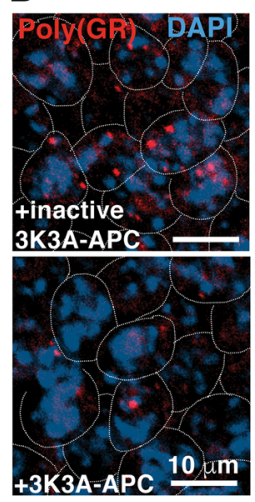

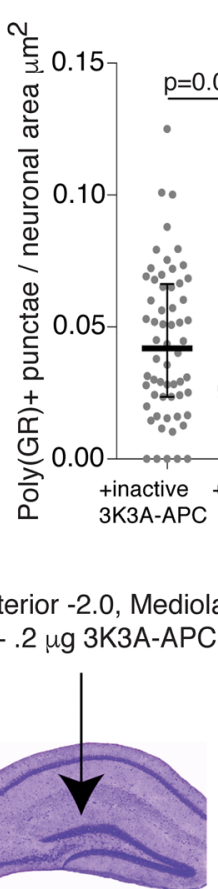

Inject at Anteroposterior -2.0, Mediolateral +1.5, Dorsoventral -1.8,

10 nmoles NMDA + .2 $\mu \mathrm{g}$ 3K3A-APC or inactive $3 \mathrm{~K} 3 \mathrm{~A}-\mathrm{APC}$ in a $.3 \mu \mathrm{l}$ volume

\section{C}

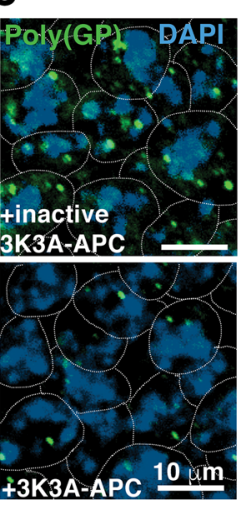

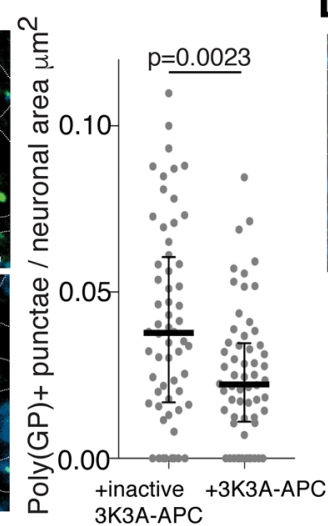

D

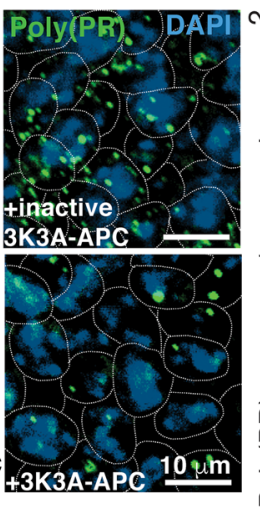

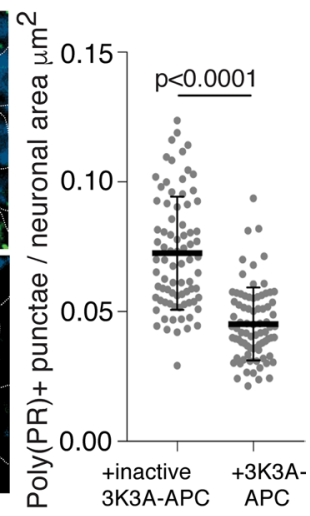

Analyze neuronal injury

by cresyl violet staining

$\mathbf{F}$

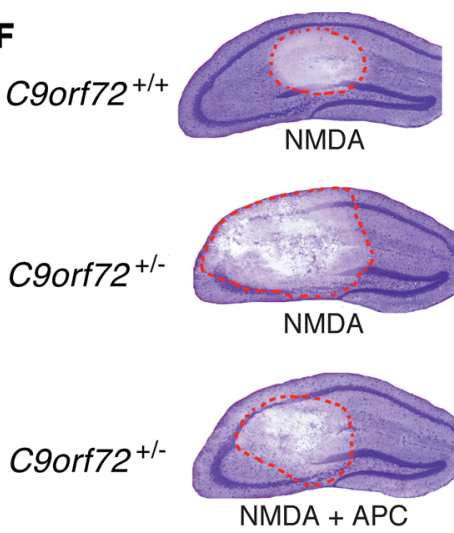

G

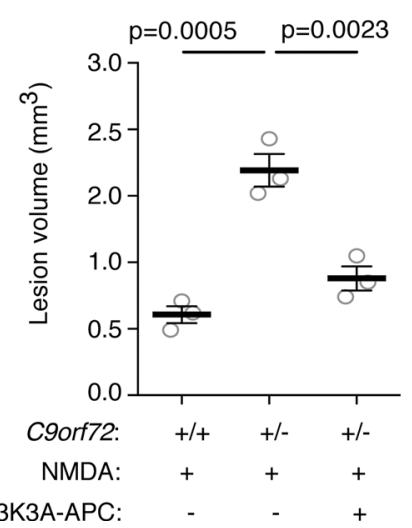

H

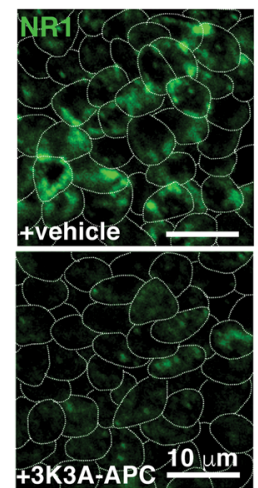

I

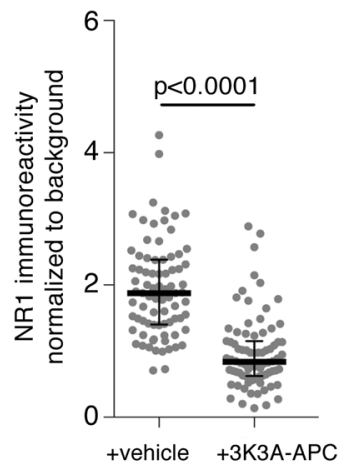

Figure 6. 3K3A-APC rescues C9ORF72 ALS proteostasis and glutamate receptor phenotypes in vivo. (A) Overview of the experimental procedure for testing the ability of 3K3A-APC to reduce DPR levels in the hippocampus of C9-BAC mice. (B-D) The effect of $10 \mathrm{nM}$ inactive 3K3A-APC or 3K3A-APC on the level of poly(GR)+ punctae in the dentate gyrus of C9-BAC mice. Mean \pm SD of the number of poly(GR)+ (B), poly(GP)+(C), and poly(PR)+ (D) punctae per cell; each data point represents a single cell. Cells quantified from 3 mice per condition, 1-way ANOVA with Tukey's correction for all comparisons. Scale bars: $10 \mu \mathrm{m}$. Dotted lines outline cell bodies. Neuronal area was determined by manual outlining in Imagej on the basis of the staining pattern provided by TUJ1 or MAP2. (E) Overview of the experimental procedure for inducing NMDA injury in the hippocampus and testing the ability of 3K3A-APC to mitigate this injury. ( $F$ and $\mathbf{G}$ ) The effect of $0.2 \mu \mathrm{g}$ of 3K3A-APC delivered in a volume of $0.3 \mu \mathrm{L}$ on NMDA-induced hippocampal injury

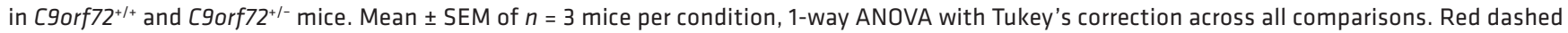
lines outline the injury sites (F). Vehicle control conditions were published in a previous study (4). (H and I) Immunostaining (H) and quantification (I) of NR1 levels in $\mathrm{Cgorf72}^{+/-}$mice treated with vehicle or $0.2 \mu \mathrm{g}$ of $3 \mathrm{~K} 3 \mathrm{~A}-\mathrm{APC}$ delivered in a volume of $0.3 \mu \mathrm{L}(n=3$ mice per condition, 72 cells quantified per condition). Each gray data point represents a single cell. Mean \pm interquartile range. Mann-Whitney test. 


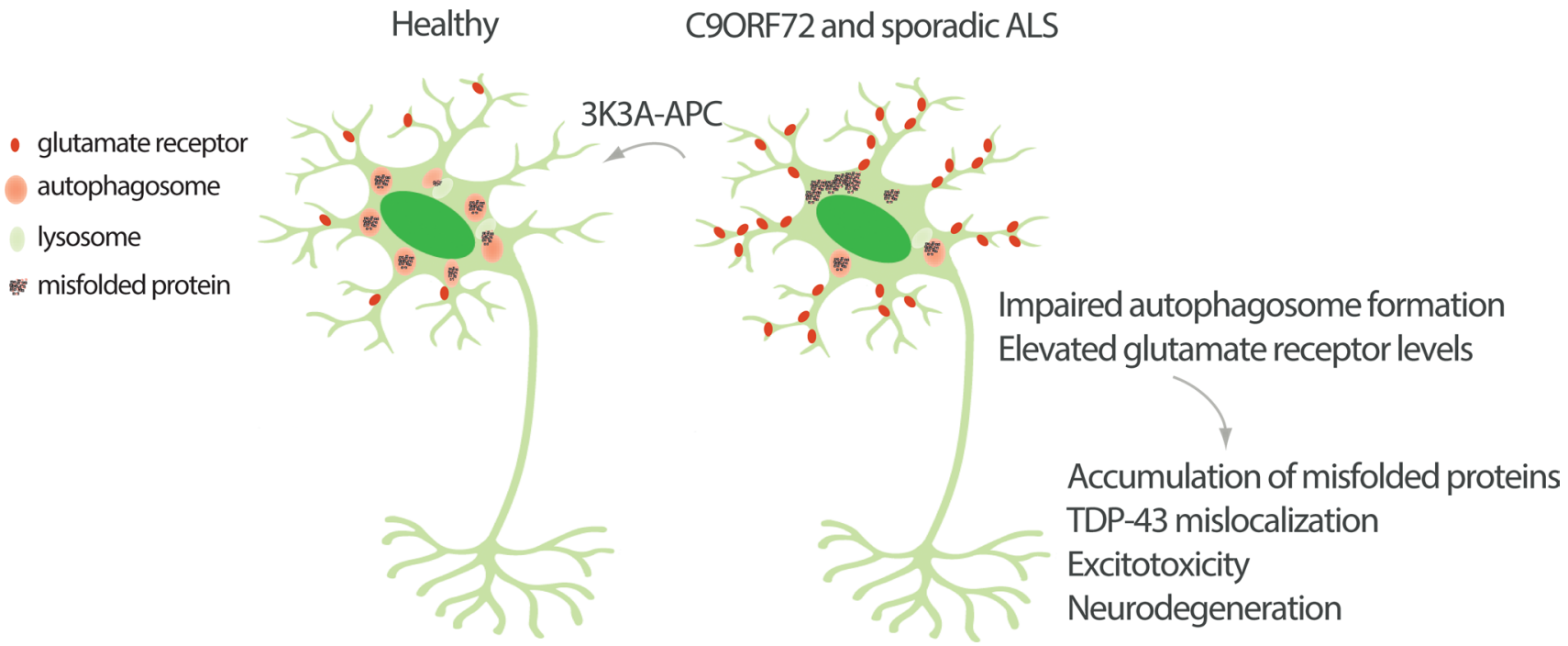

Figure 7. Model depicting the detrimental effects and therapeutic treatment of the autophagosome and glutamate receptor phenotypes shared by C9ORF72 and sporadic ALS iMNs.

and after the study and were negative. Viruses were harvested at 48 hours and 72 hours after transfection. Viral supernatants were filtered with $0.45-\mu \mathrm{m}$ filters, incubated with Lenti-X concentrator (Clontech) for 24 hours at $4^{\circ} \mathrm{C}$, and centrifuged at $1500 \mathrm{~g}$ at $4^{\circ} \mathrm{C}$ for 45 minutes. Pellets were resuspended in $300 \mu \mathrm{L}$ DMEM plus $10 \% \mathrm{FBS}$ and stored at $-80^{\circ} \mathrm{C}$.

Conversion of iPSCs into iMNs. iPSCs were tested for mycoplasma before, during, and after the study and were negative. iPSCs were first differentiated into fibroblast-like cells to enable efficient retroviral transduction. iPSCs $\left(1 \times 10^{6}\right.$ cells/flask) were seeded in a T75 flask that was coated with Matrigel (Corning) and cultured in mTeSR until reaching $70 \%$ to $80 \%$ confluence. Cells were then cultured in fibroblast medium (20\% FBS in DMEM) for 14 days. Cells were passaged 1:1 with Accutase (Innovative Cell Technologies) to another flask that was coated with Matrigel and cultured in fibroblast medium until they reached $90 \%$ confluence. Cells were then passed with $0.25 \%$ trypsin to 3 flasks that were coated with $0.1 \%$ gelatin and cultured in 10\% FBS in DMEM. Reprogramming of the fibroblast-like cells was performed in 96-well plates $\left(8 \times 10^{3}\right.$ cells/well) or 13 -mm plastic coverslips $\left(3.2 \times 10^{4}\right.$ cells/coverslip) that had been sequentially coated with gelatin $(0.1 \%, 1$ hour) and laminin ( 2 to 4 hours) at room temperature. Seven iMN factors were added in 100 to $200 \mu \mathrm{L}$ fibroblast medium per well of the 96 -well plate with $5 \mathrm{mg} / \mathrm{mL}$ polybrene. Cultures were transduced with lentivirus encoding the $H b 9:: R F P$ reporter 48 hours after transduction with transcription factor-encoding retroviruses. On day 5, primary mouse cortical glial cells from P1 ICR pups were added to the transduced cultures in glia medium containing MEM (Life Technologies), 10\% donor equine serum (HyClone), 20\% glucose (Sigma-Aldrich), and 1\% penicillin/streptomycin. On day 6, cultures were switched to N3 medium containing DMEM/F12 (Life Technologies), 2\% FBS, 1\% penicillin/streptomycin, N2 and B27 supplements (Life Technologies), $7.5 \mu \mathrm{M}$ RepSox (Selleck), and $10 \mathrm{ng} / \mathrm{mL}$ each of GDNF, BDNF, and CNTF (R\&D Systems). The iMN cultures were maintained in N3 medium, changed every other day, unless otherwise noted.

Immunocytochemistry. iMNs were fixed in $4 \%$ paraformaldehyde for 1 hour at $4^{\circ} \mathrm{C}$, permeabilized with PBS containing $0.5 \%$ Tween $20\left(0.5 \%\right.$ PBS-T) overnight at $4^{\circ} \mathrm{C}$, blocked with $10 \%$ donkey serum in $0.1 \%$ PBS-T at room temperature for 2 hours, and incubated with primary antibodies at $4^{\circ} \mathrm{C}$ overnight. Cells were then washed with $0.1 \%$ PBS-T and incubated with Alexa Fluor-conjugated secondary antibodies (Life Technologies) in blocking buffer for 2 hours at room temperature. Cells were stained with DAPI (Life Technologies) or Hoechst and then mounted on slides with Vectashield (Vector Labs). Neuronal area was determined by manual outlining in Image $(\mathrm{NIH})$ on the basis of the staining pattern provided by TUJ1 or MAP2. Images were acquired on an LSM 800 confocal microscope (Zeiss). The following primary antibodies were used: mouse anti-HB9 (Developmental Studies Hybridoma Bank, catalog 81.5C10, 1:10); mouse anti-TUJ1 (EMD Millipore, catalog AB9354, 1:1000); mouse anti-NR1 (EMD Millipore, catalog MAB363, 1:10); rabbit anti-poly(PR) (Proteintech, catalog 23979-1-AP, 1:50), rabbit anti-poly(PR) 
(Proteintech, catalog 23978-1-AP, 1:50), rabbit anti-TDP-43 (Proteintech, catalog 10782-2-AP, 1:200), and mouse anti-LAMP2 (DSHB, catalog H4B4, 1:4000).

Preparation of $3 K 3 A-A P C$. 3K3A-APC was produced as described previously (23) and was a gift from $\mathrm{ZZ}$ Biotech. Human 3K3A-Protein C (3K3A-PC) zymogen was stably transfected into Chinese hamster ovary $(\mathrm{CHO})$ cells that were grown in suspension in $\mathrm{CD}$ OptiCHO medium (Invitrogen) supplemented with $2 \mathrm{mM}$ $\mathrm{CaCl}_{2}, 10 \mu \mathrm{g} / \mathrm{mL}$ vitamin $\mathrm{K}$, and $2 \mathrm{mM}$ GlutaMAX (Invitrogen). After the required number of serial expansions of culture volumes using shake flask passages and WAVE culturing, the final stirred-tank bioreactor volume was either $200 \mathrm{~L}$ or $2500 \mathrm{~L}$ in which culture was performed for 10 days at $35^{\circ} \mathrm{C}$. After $0.2-\mu \mathrm{m}$ filtration, the clarified culture supernatant was purified using chromatography on a $\mathrm{Q}$ Sepharose fast-flow ion-exchange column and hydrophobic affinity column. The purified 3K3A-PC zymogen was then activated with recombinant human thrombin (Recothrom, Zymogenetics) to generate 3K3A-APC. Thrombin was removed from the reaction mixture using ion-exchange chromatography with a UNOsphere S flow-through resin (Bio-Rad). The concentration of 3K3A-APC was adjusted using tangential flow filtration. Following the concentration step, UNOsphere Q ion-exchange flow through was used with buffer exchange and concentration adjustment, prior to Planova filtration and addition of polysorbate-80. 3K3A-APC was characterized by SDS-PAGE under reducing and nonreducing conditions, SE-FPLC, amidolytic activity by a chromogenic assay, and optical density at $280 \mathrm{~nm}$. Inactive 3K3A-APC was generated by heat-denaturing APC.

Induced neuron survival assay. $H b 9:: \mathrm{RFP}^{+} \mathrm{iMNs}$ appeared between days 13 and 16 after retroviral transduction. RepSox was removed on day 17 and the survival assay was initiated. For the glutamate treatment condition, $10 \mu \mathrm{M}$ glutamate was added to the culture medium on day 17 and removed after 12 hours. Cells were then maintained in N3 medium with neurotrophic factors without RepSox. Cultures were treated with $10 \mathrm{nM}$ inactive or active 3K3A-APC after glutamate was removed. The inactive and active 3K3A-APC were maintained for the remainder of the experiment and replenished every other day. For treatment with rapamycin, $10 \mu \mathrm{M}$ DMSO or rapamycin (Sigma-Aldrich, R8781-200UL) was initiated after the 12-hour glutamate treatment was completed. For PAR1 and PAR2 antagonist treatment, cultures were cotreated with $3 \mu \mathrm{M}$ PAR1 or PAR2 antagonist starting after glutamate treatment. For PAR1, PAR2, and PAR3 ASO treatment, the cultures were pretreated one time with $9 \mu \mathrm{M}$ ASOs for 72 hours before the pulse glutamate treatment. PAR1 and PAR2 antagonists were purchased from Tocris (RWJ56110, catalog 2614 and AC55541, catalog 3369, respectively). PAR1, PAR2, and PAR3 ASO gapmers were designed and produced by IDT; they contained 2'-O-Me and phosphorothioate linkage modifications. Longitudinal tracking was performed by imaging neuronal cultures in a Nikon Biostation CT or Molecular Devices ImageExpress once every 24 to 72 hours starting on day 17. Tracking of neuronal survival was performed using SVcell 3.0 (DRVision Technologies) or ImageJ. Neurons were scored as dead when their soma was no longer detectable by RFP fluorescence. All neuron survival assays were performed at least twice, with equal numbers of neurons from 3 individual replicates from one of the trials being used for the quantification shown. All trials quantified were representative of other trials of the same experiment. When iMNs from multiple independent donors were combined into one survival trace in the Kaplan-Meier plots for clarity, this is noted in the figure legend.

Confocal imaging of $i M N$ s. Confocal microscopy images were acquired using a Zeiss LSM800 microscope.

Quantification of glutamate receptors. For neurites, ImageJ was used to automate detection of $\mathrm{NR} 1^{+}$punctae in a given area using a threshold of 30 .

Calcium imaging of iMNs. Calcium imaging was performed using Fluo-4-AM (Thermo Fisher Scientific, F14201) according to the manufacturer's instructions. Cell cultures were treated with $1 \mu \mathrm{M}$ cyclopiazonic acid for 30 minutes prior to the start of calcium imaging in order to deplete calcium stores from the endoplasmic reticulum and therefore enable more straightforward detection of calcium influx. At the start of the calcium imaging assay, day $17 \mathrm{iMN} /$ mixed glia cocultures were placed into N3 medium with an additional $1.5 \mu \mathrm{M}$ glutamate and at least 3 fields per culture were imaged by time lapse for 30 to 60 seconds using a Nikon Ti inverted microscope. Calcium transients per iMN were quantified manually.

$q R T-P C R$. Total RNA was extracted from sorted iMNs on day 21 after transduction with a TRIzol RNA Extraction Kit (Life Technologies) and reverse transcribed with an Oligo dT primer using a ProtoScript II First Strand Synthesis Kit (NEB). RNA integrity was checked using the Experion system (Bio-Rad). Real-time PCR was performed with iTaq Universal SYBR Green Supermix (Bio-Rad) using primers shown in Supplemental Table 3.

Generation of Dox-NIL iMNs and biotinylation of surface-bound glutamate receptors. A doxycycline-inducible (Dox-inducible) NGN2, ISL1, LHX3 (NIL) polycistronic construct was previously integrated into the AAVS1 
safe harbor locus of the C9-ALS patient iPSC line using CRISPR/Cas9 editing (4). Dox-NIL iMNs were generated by plating at approximately $25 \%$ confluence on Matrigel-coated plates and adding $1 \mathrm{mg} / \mathrm{mL}$ Dox in N3 media plus $7.5 \mu \mathrm{M}$ RepSox 1 day after plating. Mouse primary mixed glia were added to the cultures on day 6, and Dox was maintained throughout conversion. iMN cultures were harvested on day 26.

Biotinylation of plasma membrane-localized glutamate receptors was performed using the Pierce Cell Surface Protein Isolation Kit (Thermo Fisher Scientific). Briefly, Dox-NIL iMNs were incubated with $0.25 \mathrm{mg} /$ $\mathrm{mL}$ Sulfo-NHS-SS-Biotin in a cold room for 1 to 2 hours with end-to-end shaking. After quenching, cells were harvested by scraping and lysed with lysis buffer from the Pierce Cell Surface Protein Isolation Kit or M-PER mammalian protein extraction buffer (Thermo Fisher Scientific). Cell lysate was incubated with High Capacity NeutrAvidin Agarose beads (Thermo Fisher Scientific), and the bound protein was eluted in $2 \times$ SDS-PAGE sample buffer supplemented with $50 \mathrm{mM}$ DTT for 1 hour at room temperature with end-to-end rotation.

Retinoic acid/purmorphamine protocol for iPSC-motor neuron differentiation and LC3 immunoblot assay. For quantifying LC3-II and LC3-I levels, iPSC motor neurons were generated as described previously (40). Cells were used for experiments after day 21 of differentiation.

To measure the effect of $3 \mathrm{~K} 3 \mathrm{~A}-\mathrm{APC}$ on autophagosome formation, iPSC motor neuron embryoid bodies were treated with $50 \mathrm{nM}$ bafilomycin (Cayman Chemical) and $10 \mathrm{nM}$ inactive or active 3K3A-APC for 48 hours before harvesting.

Immunoblotting. For total lysate analyses relevant to glutamate receptor levels, Dox-NIL iMNs from healthy controls and ALS patients were collected on day 26 after transduction in RIPA buffer (Sigma-A1drich) with a protease inhibitor cocktail (Roche).

Protein quantity was measured by the BCA assay (Pierce) and the proteins were run in a 10\% SDS gel at $4^{\circ} \mathrm{C}$, and the proteins were transferred onto an Immobilon membrane (Millipore). The membrane was blocked with $5 \%$ milk in $0.1 \%$ PBS-T (Sigma-Aldrich), incubated with primary antibodies overnight at $4^{\circ} \mathrm{C}$, washed 3 times with $0.1 \%$ PBS-T, and then incubated with horseradish peroxidase-conjugated (HRP-conjugated) secondary antibody (Santa Cruz Biotechnology). After 3 washes with $0.1 \%$ PBS-T, blots were visualized using an Amersham ECL Western Blotting Detection Kit (GE) or the SuperSignal West Femto Maximum Sensitivity Substrate (Thermo Fisher Scientific) and developed on X-ray film (Genesee). The following primary antibodies were used: mouse anti-GAPDH (Santa Cruz Biotechnology, catalog sc-32233, 1:1000), mouse antiNR1 (Novus, catalog NB300118, 1:1000), mouse anti-transferrin receptor (Thermo Fisher Scientific, catalog 136800, 1:1000), mouse anti-TUJ1 (Biolegend, catalog MMS-435P, 1:2000), rabbit anti-poly(GR) (Proteintech, catalog 23978-1-AP, 1:1000), anti-mouse-HRP (Cell Signaling Technology, catalog 7076S, 1:5000), anti-rabbitHRP (Cell Signaling Technology, catalog 7074S, 1:5000), and anti-LC3 (VWR, catalog 101732-348, 1:1000).

RNA-Seq. C9-ALS iMNs were cultured with $10 \mathrm{nM}$ inactive 3K3A-APC or 3K3A-APC for 3 days and then $H 69:: \mathrm{RFP}^{+}$iMNs were flow purified. 3'-Digital gene expression RNA-Seq of all samples was performed by Amaryllis Nucleics. Briefly, mRNA was extracted using the NEBNext Poly(A) mRNA Magnetic Isolation Module according to the manufacturer's instructions. 3' RNA-Seq libraries were generated using the 3'-Digital Gene Expression RNAseq Library Kit (Amaryllis Nucleics). Libraries were sequenced on an Illumina NextSeq 500 sequencer. A total of 10 million to 25 million 80-bp, single-end reads were obtained for each sample. Reads were aligned to the Hg38 transcriptome using HISAT2 (41). A count table was obtained using FeatureCounts (42) with strand specificity enabled. Differential expression analysis was performed using DESeq2 (43). The DESeq2 sample description table used, count table, and DESeq2 results can be found in the supplemental data. The sequencing data have been deposited in NCBI's Gene Expression Omnibus (GEO) and can be accessed using GEO Series accession number GSE133162.

$I P A$. To determine significant pathways, differentially expressed genes for inactive $3 \mathrm{~K} 3 \mathrm{~A}-\mathrm{APC}$ versus 3K3A-APC treatment at 3 days were uploaded and analyzed by using the IPA tool (Qiagen). We focused on "Top Canonical Pathways." Only canonical pathways with $-\log (P$ value $)$ greater than 1.5 were retained. $P$ values were calculated by using Fisher's exact test.

Enrichr. KEGG pathway enrichment analysis was performed by providing Enrichr with a list of all genes identified by DESeq2 as having a greater than 95\% chance of being deferentially expressed (44).

Code availability. Code for RNA-Seq analysis can be obtained by emailing chida@usc.edu or helensfalk@gmail.com.

mRFP-GFP-LC3 assays in iMNs. Retrovirus encoded by pMXs-mRFP-GFP-LC3 was transduced into converting iMN cultures 1 day after transduction with iMN reprogramming factors. On day 17 , iMNs were treated with $50 \mathrm{nM}$ bafilomycin and $10 \mathrm{nM}$ inactive 3K3A-APC, $10 \mathrm{nM} 3 \mathrm{~K} 3 \mathrm{~A}-\mathrm{APC}, 10 \mu \mathrm{M}$ DMSO, 
or $10 \mu \mathrm{M}$ rapamycin (Sigma-Aldrich, R8781-200UL) in combinations described in the text and figure legends for 24 hours before being fixed with $4 \%$ paraformaldehyde at $4{ }^{\circ} \mathrm{C}$ for 1 hour. Motor neuron cultures were immunostained to detect HB9 levels using a mouse anti-HB9 antibody (Developmental Studies Hybridoma Bank, 1:10 dilution) at $4^{\circ} \mathrm{C}$ for 48 hours. Coverslips were imaged on a Zeiss LSM 800 confocal microscope. Quantification was performed using ImageJ.

NMDA / APC hippocampal administration and NMDA-lesion site analysis. Mice were anesthetized with i.p. ketamine $(100 \mathrm{mg} / \mathrm{kg})$ and xylazine $(10 \mathrm{mg} / \mathrm{kg})$, and body temperature kept at $36.9^{\circ} \mathrm{C} \pm 0.1^{\circ} \mathrm{C}$ with a thermostatic heating pad. Mice were placed in a stereotactic apparatus (ASI Instruments) and the head was fixed accordingly. A burr hole was drilled, and an injection needle (33 gauge) was lowered into the hippocampus between CA1 and the dentate gyrus (anteroposterior -2.0 , mediolateral +1.5 , dorsoventral -1.8). NMDA (10 nmoles) and $0.2 \mu \mathrm{g}$ of $3 \mathrm{~K} 3 \mathrm{~A}-\mathrm{APC}$ or vehicle in $0.3 \mu \mathrm{L}$ of PBS, pH 7.4 was infused over 2 minutes using a micro-injection system (World Precision Instruments).

For the DPR experiments, inactive 3K3A-APC was used as a control for any artifacts introduced into the downstream immunostaining measurements. After injection, the needle was left in place for an additional 8 minutes after the injection. Animals were euthanized 48 hours later. For injury size determination, brains were quickly removed, frozen on dry ice, and stored at $-80^{\circ} \mathrm{C}$ until processing. Coronal sections (30 $\mu \mathrm{m}$ thick) were prepared using a cryostat, and every fifth section $1 \mathrm{~mm}$ anterior and posterior to the site of injection was stained with cresyl violet. The lesion area was identified by the loss of staining, measured by ImageJ and integrated to obtain the volume of injury.

Mouse immunostaining and quantification. All animal use and care were in accordance with local institution guidelines of the University of Southern California and the IACUC board of the University of Southern California with the protocol numbers 20546 and 11938. Wild-type C57BL/6J (strain: 000664), and C9-BAC (C57BL/6J-Tg(C9orf72_i3)112Lutzy/J, strain: 023099) were purchased from Jackson Laboratories. Mice were housed in standard conditions with food and water ad libitum in the conventional vivarium at the University of Southern California.

Immunohistochemistry. C9-BAC mice (36) (>6 months old) were transcardially perfused with PBS and subsequently with $4 \%$ formaldehyde. Cryoprotection occurred in $20 \%$ sucrose. After snap freezing, tissue was sectioned by cryostat at 20- $\mu \mathrm{m}$ thickness and stained with the following primary antibodies: antipoly(GP) (catalog 24494-1-AP, ProteinTech), anti-poly(GR) (catalog MABN778, Millipore), anti-poly(PR) (catalog 23979-1-AP, ProteinTech), anti-NR1 (catalog NB300118, Novus), and anti-MAP2 (catalog ab5392, Abcam). Antigen retrieval was achieved with Target Retrieval Solution ( $\mathrm{pH}$ 9, Agilent, Dako) for the DPR staining. Images were collected using a Zeiss LSM800 confocal microscope. The scientist performing the DPR quantification was blinded to the genotype or treatment condition of the samples. Confocal microscopy images of DPR punctae were adjusted for brightness and contrast for optimal visualization of the DPR punctae; this occurred to the same extent for the vehicle- and APC-treated side of the hippocampus.

Genotyping. To provide a quantitative measure of (GGGGCC) ${ }_{n}$ hexanucleotide expansion in C9ORF72, $100 \mathrm{ng}$ of genomic DNA was amplified by touchdown PCR using primers shown in Supplemental Table 2, in a $28-\mu \mathrm{L}$ PCR reaction consisting of $0.2 \mathrm{mM}$ each of 7-deaza-2-deoxyguanine triphosphate (deaza-dGTP) (NEB), dATP, dCTP, and dTTP, 7\% DMSO, 1× Q-Solution, 1× Taq PCR buffer (Roche), $0.9 \mathrm{mM} \mathrm{MgCl}_{2}, 0.7 \mu \mathrm{M}$ reverse primer (4 GGGGCC repeats with an anchor tail), 1.4 $\mu \mathrm{M}$ 6FAM fluorescently labeled forward primer, and $1.4 \mu \mathrm{M}$ anchor primer corresponding to the anchor tail of reverse primer (Supplemental Table 2). During the PCR, the annealing temperature was gradually decreased from $70^{\circ} \mathrm{C}$ and $56^{\circ} \mathrm{C}$ in $2^{\circ} \mathrm{C}$ increments with a 3-minute extension time for each cycle. The PCR products were purified by QiaQuick PCR purification kit (Qiagen) and analyzed using an ABI3730 DNA Analyzer and Peak Scanner Software v1.0 (Life Technologies).

To detect the presence of variants in other genes in sporadic ALS lines, whole-genome sequencing was performed by the New York Genome Center, or whole-exome sequencing was performed at the Children's Hospital Los Angeles Genomics Facility. The genome/exome sequences were aligned to hg19 by Novoalign. We used an in-house tool developed by Anton Valouev (USC) for the removal of duplicate reads. Variant calling occurred by the Genome Analysis Toolkit (Broad Institute).

Statistical analysis. Analysis was performed with the statistical software package Prism Origin (GraphPad Software). Statistical analysis of iMN survival experiments was performed using a 2-sided log-rank test to account for events that did not occur (i.e., iMNs that did not degenerate before the end of the experiment). For each line, the survival data from 90 iMNs were selected randomly using Microsoft Excel, and 
these data were used to generate the survival curve. If all iMNs degenerated in a given experiment, statistical significance was calculated using a 2-tailed Student's $t$ test.

For all other experiments, analyses of iMN samples were performed with the statistical software package Prism Origin v.7.0a (GraphPad Software). The normal distribution of data sets was tested by the D'Agostino-Pearson omnibus normality test. Differences between multiple groups were analyzed using 1-way ANOVA with Tukey's correction for all comparisons, unless the data were non-normally distributed for which nonparametric Kruskal-Wallis testing was used. Mean and standard deviation or standard error of the mean was used for normally distributed data sets, and the median and interquartile range were used for non-normally distributed data sets. Differences between 2 groups were analyzed using a 2-tailed Student's $t$ test, unless the data were non-normally distributed, in which case 2-sided Mann-Whitney testing was used. Significance was assumed at $P<0.05$.

For all experiments, sample size was chosen using a power analysis based on pilot experiments that provided an estimate of effect size (http://ww.stat.ubc.ca/ rollin/stats/ssize/n2.html). Mice used for immunohistochemical analysis were selected randomly from a set of genotyped animals (genotypes were known to investigators). Mouse tissue sections used for immunohistochemical analysis were selected randomly. The sections were only not used if immunostaining failed. For iMN survival assays, iMNs from 3 biologically independent iMN conversions were used to generate the Kaplan-Meier plot shown. iMN survival times were confirmed by manual longitudinal tracking by an individual who was blinded to the identity of the genotype and condition of each sample. To select 90 iMNs per condition for analysis,more than 90 neurons were selected for tracking randomly using the images from day 1 of the assay. Subsequently, the survival values for 90 cells were selected at random using the RAND function in Microsoft Excel. For quantification of immunofluorescence, samples were quantified by an individual who was blinded to the identity of the genotype of each sample. All other quantification was performed by individuals blinded to the identity of each sample.

Study approval. All experiments involving live vertebrates (cortical glial isolation) performed at USC were done in compliance with ethical regulations approved by the USC IACUC.

\section{Author contributions}

YS, STH, BVZ, and JKI conceived the project. YS, STH, GR, SL, GRL, KAS, CS, YW, MC, JL, APS, BVZ, and JKI designed the experiments. YS, STH, GR, SL, GRL, KAS, CS, YW, MC, JL, and APS performed experiments and interpreted data. TS helped to perform whole-genome sequencing analysis on the ALS patient cell lines. STH performed RNA-Seq analysis. YS, STH, and JKI prepared the manuscript. YS and STH contributed equally to this work. All authors discussed the results and commented on the manuscript.

\section{Acknowledgments}

We thank the NINDS Biorepository at Coriell Institute for providing the cell lines used for this study. Whole-genome sequencing was provided by the University of Washington Center for Mendelian Genomics (UW-CMG) and was funded by NHGRI and NHLBI grants UM1 HG006493 and U24 HG008956 and by the Office of the Director, NIH, under award number S10OD021553. The content is solely the responsibility of the authors and does not necessarily represent the official views of the NIH. We thank Emily Wong and Anton Valouev for assistance with whole-genome sequencing and analysis. We thank Helen Falk and Sandy Falk of Ji Informatics for performing RNA-Seq analysis. We thank USC Libraries Bioinformatics Service for assisting with data analysis. We thank the Choi Family Therapeutic Screening Facility for chemical screening support and DRVision LLC for imaging support. 3K3A-APC was a gift from ZZ Biotech. This work was supported by in part by NIH grant NS090904 to BVZ and startup funds to BVZ. This work was also supported by NIH grants R00NS077435 and R01NS097850, US Department of Defense grant W81XWH-15-1-0187, and grants from the Donald E. and Delia B. Baxter Foundation, the Tau Consortium, the Frick Foundation for ALS Research, the Muscular Dystrophy Association, the New York Stem Cell Foundation, the Alzheimer's Drug Discovery Foundation, the Association for Frontotemporal Degeneration, the Pape Adams Foundation, the John Douglas French Alzheimer's Foundation, the Harrington Discovery Institute, the Merkin Family Foundation, the USC Keck School of Medicine Regenerative Medicine Initiative, the USC Broad Innovation Award, the University of Southern California Alzheimer's Disease Research Center, and the Southern California Clinical and Translational Science Institute to JKI. JKI is a New York Stem Cell Foundation-Robertson Investigator. KAS was supported in part by a Muscular Dystrophy Association development grant. 
Address correspondence to: Justin K. Ichida, 1425 San Pablo Street, Los Angeles, California 90033, USA. Phone: 323.442.0063; Email: ichida@usc.edu.

1. Pihlstrøm L, Wiethoff S, Houlden H. Genetics of neurodegenerative diseases: an overview. Handb Clin Neurol. 2017;145:309-323.

2. Menzies FM, Fleming A, Rubinsztein DC. Compromised autophagy and neurodegenerative diseases. Nat Rev Neurosci. 2015;16(6):345-357.

3. Ichida JK, Kiskinis E. Probing disorders of the nervous system using reprogramming approaches. EMBO J. 2015;34(11):1456-1477.

4. Shi Y, et al. Haploinsufficiency leads to neurodegeneration in C9ORF72 ALS/FTD human induced motor neurons. Nat Med. 2018;24(3):313-325.

5. Wen X, et al. Antisense proline-arginine RAN dipeptides linked to C9ORF72-ALS/FTD form toxic nuclear aggregates that initiate in vitro and in vivo neuronal death. Neuron. 2014;84(6):1213-1225.

6. Wainger BJ, et al. Intrinsic membrane hyperexcitability of amyotrophic lateral sclerosis patient-derived motor neurons. Cell Rep. 2014;7(1):1-11.

7. Van Damme P, Robberecht W, Van Den Bosch L. Modelling amyotrophic lateral sclerosis: progress and possibilities. Dis Model Mech. 2017;10(5):537-549

8. Kramer NJ, et al. CRISPR-Cas9 screens in human cells and primary neurons identify modifiers of C9ORF72 dipeptide-repeat-protein toxicity. Nat Genet. 2018;50(4):603-612.

9. Donnelly CJ, et al. RNA toxicity from the ALS/FTD C9ORF72 expansion is mitigated by antisense intervention. Neuron. 2013;80(2):415-428.

10. Zhang K, et al. The C9orf72 repeat expansion disrupts nucleocytoplasmic transport. Nature. 2015;525(7567):56-61.

11. Kiskinis E, et al. Pathways disrupted in human ALS motor neurons identified through genetic correction of mutant SOD1. Cell Stem Cell. 2014;14(6):781-795.

12. Moore S, et al. ADAR2 mislocalization and widespread RNA editing aberrations in C9orf72-mediated ALS/FTD. Acta Neuropathol. 2019;138(1):49-65.

13. Son EY, et al. Conversion of mouse and human fibroblasts into functional spinal motor neurons. Cell Stem Cell. 2011;9(3):205-218.

14. Neumann M, et al. Ubiquitinated TDP-43 in frontotemporal lobar degeneration and amyotrophic lateral sclerosis. Science. 2006;314(5796):130-133.

15. Barmada SJ, Skibinski G, Korb E, Rao EJ, Wu JY, Finkbeiner S. Cytoplasmic mislocalization of TDP-43 is toxic to neurons and enhanced by a mutation associated with familial amyotrophic lateral sclerosis. J Neurosci. 2010;30(2):639-649.

16. Webster CP, et al. The C9orf72 protein interacts with Rab1a and the ULK1 complex to regulate initiation of autophagy. EMBO J. 2016;35(15):1656-1676.

17. Sellier C, et al. Loss of C9ORF72 impairs autophagy and synergizes with polyQ Ataxin-2 to induce motor neuron dysfunction and cell death. EMBO J. 2016;35(12):1276-1297.

18. Kimura S, Noda T, Yoshimori T. Dissection of the autophagosome maturation process by a novel reporter protein, tandem fluorescent-tagged LC3. Autophagy. 2007;3(5):452-460.

19. Griffin JH, Zlokovic BV, Mosnier LO. Activated protein C, protease activated receptor 1, and neuroprotection. Blood. 2018;132(2):159-169.

20. Zhong Z, et al. Activated protein C therapy slows ALS-like disease in mice by transcriptionally inhibiting SOD1 in motor neurons and microglia cells. J Clin Invest. 2009;119(11):3437-3449.

21. Yen YT, et al. Enhancing autophagy with activated protein $C$ and rapamycin protects against sepsis-induced acute lung injury. Surgery. 2013;153(5):689-698

22. Mosnier LO, Gale AJ, Yegneswaran S, Griffin JH. Activated protein C variants with normal cytoprotective but reduced anticoagulant activity. Blood. 2004;104(6):1740-1744.

23. Williams PD, Zlokovic BV, Griffin JH, Pryor KE, Davis TP. Preclinical safety and pharmacokinetic profile of 3K3A-APC, a novel, modified activated protein C for ischemic stroke. Curr Pharm Des. 2012;18(27):4215-4222.

24. Lyden P, et al. Phase 1 safety, tolerability and pharmacokinetics of 3K3A-APC in healthy adult volunteers. Curr Pharm Des. 2013;19(42):7479-7485.

25. Yamaguchi M, et al. Structural insights into Atg10-mediated formation of the autophagy-essential Atg12-Atg5 conjugate. Structure. 2012;20(7):1244-1254

26. Barmada SJ, et al. Autophagy induction enhances TDP43 turnover and survival in neuronal ALS models. Nat Chem Biol. 2014;10(8):677-685.

27. Devlin AC, et al. Human iPSC-derived motoneurons harbouring TARDBP or C9ORF72 ALS mutations are dysfunctional despite maintaining viability. Nat Commun. 2015;6:5999.

28. Kuo JJ, et al. Hyperexcitability of cultured spinal motoneurons from presymptomatic ALS mice. J Neurophysiol. 2004;91(1):571-575.

29. van Zundert B, et al. Neonatal neuronal circuitry shows hyperexcitable disturbance in a mouse model of the adult-onset neurodegenerative disease amyotrophic lateral sclerosis. J Neurosci. 2008;28(43):10864-10874.

30. Vucic S, Kiernan MC. Novel threshold tracking techniques suggest that cortical hyperexcitability is an early feature of motor neuron disease. Brain. 2006;129(Pt 9):2436-2446.

31. Vucic S, Kiernan MC. Axonal excitability properties in amyotrophic lateral sclerosis. Clin Neurophysiol. 2006;117(7):1458-1466.

32. Rothstein JD, et al. Knockout of glutamate transporters reveals a major role for astroglial transport in excitotoxicity and clearance of glutamate. Neuron. 1996;16(3):675-686.

33. Chen P, Gu Z, Liu W, Yan Z. Glycogen synthase kinase 3 regulates N-methyl-D-aspartate receptor channel trafficking and function in cortical neurons. Mol Pharmacol. 2007;72(1):40-51.

34. Seebohm G, et al. Identification of a novel signaling pathway and its relevance for GluA1 recycling. PLoS ONE. 2012;7(3):e33889.

35. Zhang Y, et al. An RNA-sequencing transcriptome and splicing database of glia, neurons, and vascular cells of the cerebral cortex. 
J Neurosci. 2014;34(36):11929-11947.

36. O'Rourke JG, et al. C9orf72 BAC transgenic mice display typical pathologic features of ALS/FTD. Neuron. 2015;88(5):892-901.

37. Krieger C, Wagey R, Shaw C. Amyotrophic lateral sclerosis: quantitative autoradiography of [3H]MK-801/NMDA binding sites in spinal cord. Neurosci Lett. 1993;159(1-2):191-194.

38. Vanhauwaert R, et al. The SAC1 domain in synaptojanin is required for autophagosome maturation at presynaptic terminals. EMBO J. 2017;36(10):1392-1411.

39. Okita K, et al. An efficient nonviral method to generate integration-free human-induced pluripotent stem cells from cord blood and peripheral blood cells. Stem Cells. 2013;31(3):458-466.

40. Du ZW, et al. Generation and expansion of highly pure motor neuron progenitors from human pluripotent stem cells. Nat Commun. 2015;6:6626.

41. Kim D, Langmead B, Salzberg SL. HISAT: a fast spliced aligner with low memory requirements. Nat Methods. 2015;12(4):357-360

42. Liao Y, Smyth GK, Shi W. featureCounts: an efficient general purpose program for assigning sequence reads to genomic features. Bioinformatics. 2014;30(7):923-930.

43. Love MI, Huber W, Anders S. Moderated estimation of fold change and dispersion for RNA-seq data with DESeq2. Genome Biol. 2014;15(12):550

44. Kuleshov MV, et al. Enrichr: a comprehensive gene set enrichment analysis web server 2016 update. Nucleic Acids Res. 2016;44(W1):W90-W97. 